\title{
Design and synthesis of novel diamide derivatives of glycine as anti- hyperglycemic agents
}

Radhika Sharma and Shubhangi S Soman*

Department of Chemistry, Faculty of Science, The M. S. University of Baroda, Vadodara, 390002 , India.

*Corresponding Author's e-mail address: shubhangiss@ rediffmail.com

Telephone number: +912562795552

First Author's e-mail address: radhika.naudiyal@gmail.com

\section{Experimental}

Reagent grade chemicals and solvents were purchased from commercial supplier and used after purification. TLC was performed on silica gel F254 plates (Merck). Merck silica gel (60-120 mesh) was used for column chromatographic purification. All reactions were carried out in nitrogen atmosphere. Melting points are uncorrected and were measured in open capillary tubes, using a Rolex melting point apparatus. IR spectra were recorded as $\mathrm{KBr}$ pellets on Perkin Elmer RX 1 spectrometer and the wave numbers reported in $\mathrm{cm}^{-1} .{ }^{1} \mathrm{H}$ NMR and ${ }^{13} \mathrm{C} \mathrm{NMR}$ spectral data were recorded on Advance Bruker 400 spectrometer $\left({ }^{1} \mathrm{H} 400 \mathrm{MHz} /{ }^{13} \mathrm{C} 100 \mathrm{MHz}\right)$ with $\mathrm{CDCl}_{3}$ or DMSO- $\mathrm{d}_{6}$ as solvent and TMS as internal standard and reported in $\delta(\mathrm{ppm}) . J$ values are in Hz. Mass spectra were determined by ESI-MS, using a Shimadzu LCMS 2020 apparatus. Elemental analyses were recorded on ThermoFinnigan Flash 11-12 series EA. All reactions were carried out under nitrogen atmosphere. Purity of all the tested compounds was greater than $97 \%$. 


\section{General procedure for the preparation of compounds (2a-k)}

A mixture of boc-glycine 1 (1.11 mmol), 1-ethyl-3-(3-dimethylaminopropyl)carbodiimide hydrochloride (EDCI) (1.67 mmol), 1-hydroxybenzotriazole (HOBt) (1.11 mmol), 4dimethylaminopyridine (DMAP) $(1.34 \mathrm{mmol})$ and amine $\left(1^{0}\right.$ and $\left.2^{0}\right)(1.22 \mathrm{mmol})$ in dichloromethane $(\mathrm{DCM})(50 \mathrm{~mL})$ was stirred at room temperature for 16 hours. The reaction was monitored using TLC. On completion of the reaction, it was washed with water $(2 \mathrm{X} 20$ $\mathrm{mL})$, brine (1X10 mL), dried over anhydrous sodium sulphate and the solvent evaporated under reduced pressure to give the crude product which was then purified by column chromatography using silica gel as stationary phase and methanol:dichloromethane (5:95) as eluent to yield desired $N$-boc glycine amide $\mathbf{2 a - k}$, as white solid.

\section{1-(phenylsulfonyl)piperidine-3-carboxylic acid (5)}

To a mixture of piperidine-3-carboxylic acid $4(1.0 \mathrm{mmol})$ and sodium carbonate $(3.0 \mathrm{mmol})$ in $25 \mathrm{~mL}$ DCM:water (1:1) benzene sulfonyl chloride (1.1 mmol) was added and the reaction mixture stirred at room temperature for 16 hours or till the completion of reaction, as monitored by TLC. On completion of reaction, the reaction mixture was washed with petroleum ether (20 $\mathrm{mL}$ ) and then acidified with conc. $\mathrm{HCl}$, till $\mathrm{pH}$ 2. The white solid thus separated was filtered, washed with water several times and then dried to yield the desired product as white solid. Yield: 91\%; white solid; m.p.: $115-117^{\circ} \mathrm{C}$; IR (KBr): 2940, 1693, $1352 \mathrm{~cm}^{-1}$; ${ }^{1} \mathrm{H}$ NMR (400 $\left.\mathrm{MHz}, \mathrm{CDCl}_{3}\right): \delta 1.41-1.50(\mathrm{~m}, 1 \mathrm{H}), 1.65-1.73(\mathrm{~m}, 1 \mathrm{H}), 1.80-1.85(\mathrm{~m}, 1 \mathrm{H}), 1.99-2.04(\mathrm{~m}, 1 \mathrm{H})$, $2.41\left(\mathrm{dt}, 1 \mathrm{H}, J_{1}=2.8 \mathrm{~Hz}, J_{2}=11.2 \mathrm{~Hz}\right), 2.57(\mathrm{t}, 1 \mathrm{H}, J=10.8 \mathrm{~Hz}), 2.65-2.71(\mathrm{~m}, 1 \mathrm{H}), 3.59(\mathrm{br}$ $\mathrm{d}, 1 \mathrm{H}, J=11.6 \mathrm{~Hz}), 3.83\left(\mathrm{dd}, 1 \mathrm{H}, J_{l}=3.2 \mathrm{~Hz}, J_{2}=7.2 \mathrm{~Hz}\right), 7.54-7.58(\mathrm{~m}, 2 \mathrm{H}), 7.61-7.63(\mathrm{~m}$, 1H), 7.77-7.79 (m, 2H), 8.98 (br s, $\left.1 \mathrm{H}) ;{ }^{13} \mathrm{C} \mathrm{NMR} \mathrm{(100} \mathrm{MHz,} \mathrm{CDCl}_{3}\right): \delta 23.88,26.21,40.73$, $46.26,47.35,127.62,129.18,132.94,135.96,178.63$. 


\section{General procedure for the preparation of compounds (6a-k)}

Compounds 2a-k were de-protected by stirring in 10\% trifluoroacetic acid (TFA) in DCM. On completion of the reaction after an hour or as monitored by TLC, the solvent was evaporated under reduced pressure and once again the product was dissolved in DCM to give solution of compounds 3a-k. To a solution of compound 5 (1.0 mmol) in DCM (20 mL), EDCI (1.5 mmol), HOBt $(1.0 \mathrm{mmol})$ and DMAP $(1.0 \mathrm{mmol})$ were added at $0-5{ }^{\circ} \mathrm{C}$, followed by the solution of compound 3a-k in DCM (5 mL) and the reaction mixture was then stirred at room temperature for 10 hours or till the completion of the reaction as detected by TLC. After completion of the reaction, it was washed with water $(2 \mathrm{X} 20 \mathrm{~mL})$, brine $(1 \mathrm{X} 10 \mathrm{~mL})$, dried over anhydrous sodium sulphate and the solvent evaporated under reduced pressure to give the crude product which was then purified by column chromatography using silica gel, employing ethylacetate:petroleum ether (70:30) as eluent to give pure product 6a-k as white solid.

N-(2-(4-fluorophenylamino)-2-oxoethyl)-1-(phenylsulfonyl)piperidine-3-carboxamide (6a). Yield: 70\%; white solid; m.p.: 196-198 ${ }^{\circ} \mathrm{C}$; IR (KBr): 3324, 3298, 2967, 2934, 2843, 2865 , 1677, 1654, 1643, $1355 \mathrm{~cm}^{-1} ;{ }^{1} \mathrm{H}$ NMR (400 MHz, DMSO-d 6 ): $\delta$ 1.23-1.27 (m, 1H), 1.46-1.49 (m, 1H), 1.72-1.79 (m, 2H), 2.10-2.20 (m, 2H), 2.50-2.51 (m, 1H), 3.58-3.63 (m, 1H), 3.66$3.72(\mathrm{~m}, 1 \mathrm{H}), 3.82-3.85(\mathrm{~m}, 2 \mathrm{H}), 7.15(\mathrm{t}, 2 \mathrm{H}, J=8.8 \mathrm{~Hz}), 7.56-7.60(\mathrm{~m}, 2 \mathrm{H}), 7.66(\mathrm{t}, 2 \mathrm{H}, J=$ 8.4 Hz), 7.72-7.74 (m, 3H), 8.39 (br s, $1 \mathrm{H}), 10.05$ (s, 1H); ${ }^{13} \mathrm{C}$ NMR (100 MHz, DMSO-d 6 ): $\delta$ $24.14,27.00,41.93,42.85,46.52,48.62,115.68,115.90,121.31,121.39,127.85,129.94$, 133.70, 135.59, 135.67, 157.23, 159.61, 168.00, 173.16; Anal. Calc. for $\mathrm{C}_{20} \mathrm{H}_{22} \mathrm{FN}_{3} \mathrm{O}_{4} \mathrm{~S}$ : C, 57.27; H, 5.29; N, 10.02; found: C, 57.42; H, 4.90; N, 9.97\%; ESI-MS: m/z $420.0[\mathrm{M}+\mathrm{H}]^{+}$. N-(2-(4-chlorophenylamino)-2-oxoethyl)-1-(phenylsulfonyl)piperidine-3-carboxamide (6b). Yield: 55\%; white solid; m.p.: 178-180 ${ }^{\circ} \mathrm{C}$; IR (KBr): 3329, 3303, 2931, 2863, 2843, 1679, 1644, 1614, 1355, $1334 \mathrm{~cm}^{-1} ;{ }^{1} \mathrm{H}$ NMR (400 MHz, DMSO-d $): \delta 1.22-1.27$ (m, 1H), 1.46-1.49 
(m, 1H), $1.75(\mathrm{t}, 2 \mathrm{H}, J=15 \mathrm{~Hz}), 2.10-2.20(\mathrm{~m}, 2 \mathrm{H}), 2.50-2.54(\mathrm{~m}, 1 \mathrm{H}), 3.60-3.69(\mathrm{~m}, 2 \mathrm{H})$, 3.84-3.86 (m, 2H), $7.36(\mathrm{~d}, 2 \mathrm{H}, J=8.8 \mathrm{~Hz}), 7.59-7.67(\mathrm{~m}, 4 \mathrm{H}), 7.72-7.74(\mathrm{~m}, 3 \mathrm{H}), 8.41(\mathrm{t}, 1 \mathrm{H}$, $J=8.0 \mathrm{~Hz}), 10.14(\mathrm{~s}, 1 \mathrm{H}) ;{ }^{13} \mathrm{C}$ NMR $\left(100 \mathrm{MHz}, \mathrm{DMSO}-\mathrm{d}_{6}\right): \delta 24.14,27.01,41.93,42.95$, $46.52,48.63,121.10,127.25,127.85,129.13,129.93,133.68,135.62,138.25,168.26,173.16$; Anal. Calc. for $\mathrm{C}_{20} \mathrm{H}_{22} \mathrm{ClN}_{3} \mathrm{O}_{4} \mathrm{~S}$ : C, 55.11; H, 5.09; N, 9.64; found: C, 55.22; H, 5.01; N, 9.58\%; ESI-MS: $m / z, 435.9[\mathrm{M}+\mathrm{H}]^{+}$.

Yield: 85\%; white solid; m.p.: 194-196 ${ }^{\circ} \mathrm{C}$; IR (KBr): 3331, 3327, 2956, 2929, 2846, 1678, 1657, 1644, 1358, $1332 \mathrm{~cm}^{-1} ;{ }^{1} \mathrm{H}$ NMR (400 MHz, DMSO-d $\left.{ }_{6}\right): \delta 1.23-1.27$ (m, 1H), 1.46-1.49 $(\mathrm{m}, 1 \mathrm{H}), 1.72-1.79(\mathrm{~m}, 2 \mathrm{H}), 2.10-2.20(\mathrm{~m}, 2 \mathrm{H}), 2.24(\mathrm{~s}, 3 \mathrm{H}), 2.50-2.56(\mathrm{~m}, 1 \mathrm{H}), 3.66-3.69(\mathrm{~m}$, 2H), 3.77-3.85 (m, 2H), $7.10(\mathrm{~d}, 2 \mathrm{H}, J=8.0 \mathrm{~Hz}), 7.45(\mathrm{~d}, 2 \mathrm{H}, J=8.0 \mathrm{~Hz}), 7.64-7.68(\mathrm{~m}, 2 \mathrm{H})$, 7.72-7.75 (m, 3H), $8.36(\mathrm{~m}, 1 \mathrm{H}), 9.90(\mathrm{~s}, 1 \mathrm{H}) ;{ }^{13} \mathrm{C}$ NMR (100 MHz, DMSO-d 6 ): $\delta 20.89,24.15$, $27.01,41.94,42.87,46.53,48.64,119.58,127.86,129.59,129.94,132.64,133.69,135.60$, 136.76, 167.80, 173.10; Anal. Calc. for $\mathrm{C}_{21} \mathrm{H}_{25} \mathrm{~N}_{3} \mathrm{O}_{4} \mathrm{~S}$ : C, 60.70; H, 6.06; N, 10.11; found: C, 60.78; H, 6.18; N, 9.90\%; ESI-MS: $m / z, 416.1[\mathrm{M}+\mathrm{H}]^{+}$.

N-(2-(3-fluorophenylamino)-2-oxoethyl)-1-(phenylsulfonyl)piperidine-3-carboxamide (6d). Yield: 62\%; white solid; m.p.: 186-188 ${ }^{\circ} \mathrm{C}$; IR (KBr): 3308, 3104, 2930, 2851, 1709, 1670 , 1616, 1351, $1317 \mathrm{~cm}^{-1}$; ${ }^{1} \mathrm{H}$ NMR (400 MHz,DMSO-d 6$): \delta$ 1.23-1.27 (m, 1H), 1.46-1.49 (m, $1 \mathrm{H}), 1.72-1.79(\mathrm{~m}, 2 \mathrm{H}), 2.10-2.20(\mathrm{~m}, 2 \mathrm{H}), 2.50-2.51(\mathrm{~m}, 1 \mathrm{H}), 3.61(\mathrm{~d}, 1 \mathrm{H}, J=10.8 \mathrm{~Hz}), 3.68$ $(\mathrm{d}, 1 \mathrm{H}, J=10.8 \mathrm{~Hz}), 3.84-3.87(\mathrm{~m}, 2 \mathrm{H}), 6.88-6.90(\mathrm{~m}, 1 \mathrm{H}), 7.26-7.36(\mathrm{~m}, 2 \mathrm{H}), 7.55-7.75(\mathrm{~m}$, 6h), $8.41\left(\right.$ br s, 1H), $10.22(\mathrm{~s}, 1 \mathrm{H}) ;{ }^{13} \mathrm{C}$ NMR (100 MHz, DMSO-d 6 ): $\delta 24.14,27.00,41.92$, $42.97,46.52,48.62,106.18,106.44,110.08,110.29,115.27,127.86,129.93,130.85,130.95$, 133.69, 135.61, 140.95, 141.06, 161.38, 163.78, 168.49, 173.17; Anal. Calc. for $\mathrm{C}_{20} \mathrm{H}_{22} \mathrm{FN}_{3} \mathrm{O}_{4} \mathrm{~S}: \mathrm{C}, 57.27 ; \mathrm{H}, 5.29 ; \mathrm{N}, 10.02$; found: C, 57.42; H, 4.90; N, 9.97\%. ESI-MS: $\mathrm{m} / z$ $420.2[\mathrm{M}+\mathrm{H}]^{+}$ 
N-(2-(3-chlorophenylamino)-2-oxoethyl)-1-(phenylsulfonyl)piperidine-3-carboxamide (6e).

Yield: 59\%; white solid; m.p.: 140-142 ${ }^{\circ} \mathrm{C}$; IR (KBr): 3412, 3303, 2948, 2843,1692, 1666, 1650, 1350, $1333 \mathrm{~cm}^{-1} ;{ }^{1} \mathrm{H}$ NMR (400 MHz, $\left.\mathrm{CDCl}_{3}\right): \delta 1.67-1.71(\mathrm{~m}, 1 \mathrm{H}), 1.75(\mathrm{~s}, 1 \mathrm{H}), 1.82-$ $1.91(\mathrm{~m}, 2 \mathrm{H}), 2.45-2.55(\mathrm{~m}, 1 \mathrm{H}), 2.64-2.74(\mathrm{~m}, 2 \mathrm{H}), 3.57(\mathrm{~d}, 1 \mathrm{H}, J=11.6 \mathrm{~Hz}), 3.74(\mathrm{~d}, 1 \mathrm{H}, J=$ $9.2 \mathrm{~Hz}), 4.14(\mathrm{~d}, 2 \mathrm{H}, J=5.2 \mathrm{~Hz}), 7.01-7.10(\mathrm{~m}, 1 \mathrm{H}), 7.16-7.24(\mathrm{~m}, 2 \mathrm{H}), 7.40-7.42(\mathrm{~m}, 1 \mathrm{H})$, 7.52-7.56 (m, 2H), 7.60-7.64 (m, 2H), 7.75-7.77 (m, 2H), $8.82(\mathrm{~s}, 1 \mathrm{H}) ;{ }^{13} \mathrm{C}$ NMR $(100 \mathrm{MHz}$, $\left.\mathrm{CDCl}_{3}\right): \delta 23.76,26.90,42.39,44.34,46.35,48.22,117.84,119.92,124.44,127.56,129.25$, 130.03, 133.10, 134.51, 135.59, 138.78, 167.04, 173.90; Anal. Calc. for $\mathrm{C}_{20} \mathrm{H}_{22} \mathrm{ClN}_{3} \mathrm{O}_{4} \mathrm{~S}: \mathrm{C}$, 55.11; H, 5.09; N, 9.64; found: C, 55.26; H, 4.98; N, 9.52\%; ESI-MS: $m / z, 436.00[\mathrm{M}+\mathrm{H}]^{+}$.

N-(2-(2-chlorophenylamino)-2-oxoethyl)-1-(phenylsulfonyl)piperidine-3-carboxamide (6f). Yield: 48\%; white solid; m.p.: 162-164 ${ }^{\circ} \mathrm{C}$; IR (KBr): 3373, 3257, 2953, 2936, 2863, 1707 , 1649, 1583, 1386, $1323 \mathrm{~cm}^{-1} ;{ }^{1} \mathrm{H}$ NMR (400 MHz, $\left.\mathrm{CDCl}_{3}\right): \delta 1.58-1.76(\mathrm{~m}, 2 \mathrm{H}), 1.80-1.93(\mathrm{~m}$, 2H), 2.41-2.48 (m, 1H), 2.63-2.70 (m, 2H), 3.61-3.64 (m, 1H), 3.75-3.82 (m, 1H), 4.13-4.14 (m, 2H), 7.01-7.10 (m, 2H), 7.26-7.30 (m, 1H), 7.36-7.39 (m, 1H), 7.53-7.57 (m, 2H), 7.61$7.65(\mathrm{~m}, 1 \mathrm{H}), 7.76-7.78(\mathrm{~m}, 2 \mathrm{H}), 8.30-8.35(\mathrm{~m}, 2 \mathrm{H}) ;{ }^{13} \mathrm{C} \mathrm{NMR}\left(100 \mathrm{MHz}, \mathrm{CDCl}_{3}\right): \delta 23.84$, $26.93,42.59,44.53,46.33,48.21,121.86,123.18,125.16,127.61,127.75,129.17,129.25$, 133.07, 134.06, 135.54, 167.23, 173.79; Anal. Calc. for $\mathrm{C}_{20} \mathrm{H}_{22} \mathrm{ClN}_{3} \mathrm{O}_{4} \mathrm{~S}: \mathrm{C}, 55.11 ; \mathrm{H}, 5.09$; N, 9.64; found: C, 55.25; H, 5.12; N, 9.58\%; ESI-MS: $m / z 436.05[\mathrm{M}+\mathrm{H}]^{+}$.

\section{N-(2-(dimethylamino)-2-oxoethyl)-1-(phenylsulfonyl)piperidine-3-carboxamide (6g).}

Yield: 80\%; white solid; m.p.: 128-130 ${ }^{\circ} \mathrm{C}$; IR (KBr): 3351, 3274, 2978, 2940, 1720, 1677 , 1635, 1365, $1338 \mathrm{~cm}^{-1} ;{ }^{1} \mathrm{H}$ NMR (400 MHz, $\left.\mathrm{CDCl}_{3}\right): \delta 1.46-1.50(\mathrm{~m}, 1 \mathrm{H}), 1.65-1.85(\mathrm{~m}, 2 \mathrm{H})$, 1.89-1.93 (m, 1H), 2.28-2.35 (m, 1H), 2.48-2.60 (m, 2H), $3.02(\mathrm{~d}, 6 \mathrm{H}, J=9.6 \mathrm{~Hz}), 3.73(\mathrm{~d}, 1 \mathrm{H}$, $J=11.6 \mathrm{~Hz}), 3.85(\mathrm{~d}, 1 \mathrm{H}, J=9.6 \mathrm{~Hz}), 4.03(\mathrm{~d}, 2 \mathrm{H}, J=8.4 \mathrm{~Hz}), 6.82(\mathrm{br} \mathrm{s}, 1 \mathrm{H}), 7.53-7.57(\mathrm{~m}$, 2H), 7.60-7.64 (m, 1H), $7.77(\mathrm{~d}, 2 \mathrm{H}, J=7.2 \mathrm{~Hz}) ;{ }^{13} \mathrm{C} \mathrm{NMR}\left(100 \mathrm{MHz}, \mathrm{CDCl}_{3}\right): \delta 24.02,27.16$, 
35.68, 35.95, 41.22, 42.84, 46.26, 48.24, 127.66, 129.13, 132.85, 135.92, 167.65, 172.54; Anal. Calc. for $\mathrm{C}_{16} \mathrm{H}_{23} \mathrm{~N}_{3} \mathrm{O}_{4} \mathrm{~S}$ : C, 54.37; H, 6.56; N, 11.89; found: C, 54.45; H, 6.62; N, 11.81\%; ESIMS: $m / z 354.0[\mathrm{M}+\mathrm{H}]^{+}$.

N-(2-oxo-2-(pyrrolidin-1-yl)ethyl)-1-(phenylsulfonyl)piperidine-3-carboxamide (6h). Yield: 66\%; white solid; m.p.: $138-140{ }^{\circ}$ C; IR (KBr): 3383, 3304, 2949, 2870, 1681, 1651, $1398 \mathrm{~cm}^{-}$ ${ }^{1} ;{ }^{1} \mathrm{H}$ NMR (400 MHz, $\left.\mathrm{CDCl}_{3}\right): \delta 1.43-1.53(\mathrm{~m}, 1 \mathrm{H}), 1.66-1.73(\mathrm{~m}, 1 \mathrm{H}), 1.79-1.84(\mathrm{~m}, 2 \mathrm{H})$, 1.90-1.94 (m, 2H), 1.98-2.03 (m, 2H), 2.27-2.33 (m, 1H), $2.49(\mathrm{t}, 1 \mathrm{H}, J=10.4 \mathrm{~Hz}) 2.55-2.60$ (m, 1H), $3.39(\mathrm{t}, 2 \mathrm{H}, J=6.8 \mathrm{~Hz}), 3.53(\mathrm{t}, 2 \mathrm{H}, J=6.8 \mathrm{~Hz}), 3.84-3.85(\mathrm{~m}, 1 \mathrm{H}), 3.86-3.87(\mathrm{~m}$, 1H), 3.95-3.99 (m, 2H), 6.86 (br s, 1H), 7.52-7.56 (m, 2H), 7.59-7.63 (m, 1H), 7.75-7.78 (m, $2 \mathrm{H}) ;{ }^{13} \mathrm{C} \mathrm{NMR}\left(100 \mathrm{MHz}, \mathrm{CDCl}_{3}\right): \delta 24.07,24.14,25.89,27.04,41.96,42.66,45.53,46.13$, 46.26, 48.36, 127.62, 129.11, 132.82, 135.88, 166.33, 172.73; Anal. Calc. for $\mathrm{C}_{18} \mathrm{H}_{25} \mathrm{~N}_{3} \mathrm{O}_{4} \mathrm{~S}$ : C, 56.97; H, 6.64; N, 11.07; found: C, 57.00; H, 6.82; N, 11.30\%; ESI-MS: m/z $380.1[\mathrm{M}+\mathrm{H}]^{+}$.

Yield: 79\%; white solid; m.p.: 178-180 ${ }^{\circ} \mathrm{C}$; IR (KBr): 3311, 2963, 2922, 2856, 1670, 1655 , 1640, 1351, $1332 \mathrm{~cm}^{-1} ;{ }^{1} \mathrm{H} \mathrm{NMR}\left(400 \mathrm{MHz}, \mathrm{CDCl}_{3}\right): \delta 1.49-1.56(\mathrm{~m}, 1 \mathrm{H}), 1.66-1.74(\mathrm{~m}, 1 \mathrm{H})$, 1.80-1.91 (m, 2H), 2.33-2.39 (m, 1H), 2.52-2.58 (m, 2H), 3.41-3.44 (m, 2H), 3.66-3.74 (m, 7H), $3.81(\mathrm{~d}, 1 \mathrm{H}, J=8.0 \mathrm{~Hz}), 4.03-4.10(\mathrm{~m}, 2 \mathrm{H}), 6.81(\mathrm{br} \mathrm{s}, 1 \mathrm{H}), 7.53-7.57$ (m, 2H), 7.60-7.64 (m, 1H), 7.76-7.78 (m, 2H); $\left.{ }^{13} \mathrm{C} \mathrm{NMR} \mathrm{(100} \mathrm{MHz,} \mathrm{CDCl}_{3}\right): \delta 23.95,27.11,41.04,42.34,42.77$, 44.78, 46.28, 48.19, 66.31, 66.69, 127.65, 129.14, 132.87, 135.89, 166.39, 172.62; Anal. Calc. for $\mathrm{C}_{18} \mathrm{H}_{25} \mathrm{~N}_{3} \mathrm{O}_{5} \mathrm{~S}: \mathrm{C}, 54.67 ; \mathrm{H}, 6.37 ; \mathrm{N}, 10.63$; found: $\mathrm{C}, 54.75 ; \mathrm{H}, 5.86 ; \mathrm{N}, 10.32 \%$; ESI-MS: $m / z 396.1[\mathrm{M}+\mathrm{H}]^{+}$.

N-(2-(1,2,3,4-tetrahydroisoquinolin-2(1H)-yl)-2-oxoethyl)-1-(phenylsulfonyl)piperidine-3carboxamide (6j). Yield: 62\%; white solid; m.p.: 142-144 ${ }^{\circ} \mathrm{C}$; IR (KBr): 3322, 2948, 2913, 2849, 1664, 1650, 1635, 1351, $1343 \mathrm{~cm}^{-1} ;{ }^{1} \mathrm{H}$ NMR (400 MHz, $\left.\mathrm{CDCl}_{3}\right): \delta 1.48-1.51(\mathrm{~m}, 1 \mathrm{H})$, 
$1.67-1.70(\mathrm{~m}, 1 \mathrm{H}), 1.80-1.85(\mathrm{~m}, 1 \mathrm{H}), 1.90-1.93(\mathrm{~m}, 1 \mathrm{H}), 2.30-2.36(\mathrm{~m}, 1 \mathrm{H}), 2.50-2.60(\mathrm{~m}$, 2H), 2.90-2.96 (m, 2H), $3.64(\mathrm{t}, 1 \mathrm{H}, J=6.0 \mathrm{~Hz}), 3.72(\mathrm{~d}, 1 \mathrm{H}, J=11.2 \mathrm{~Hz}), 3.83-3.89(\mathrm{~m}, 2 \mathrm{H})$, 4.11-4.16 (m, 2H), $4.57(\mathrm{~s}, 1 \mathrm{H}), 4.78(\mathrm{~s}, 1 \mathrm{H}), 7.11-7.13(\mathrm{~m}, 1 \mathrm{H}), 7.16-7.20(\mathrm{~m}, 2 \mathrm{H}), 7.22-7.25$ $(\mathrm{m}, 2 \mathrm{H}), 7.53-7.57(\mathrm{~m}, 2 \mathrm{H}), 7.60-7.64(\mathrm{~m}, 1 \mathrm{H}), 7.76-7.78(\mathrm{~m}, 2 \mathrm{H}) ;{ }^{13} \mathrm{C}$ NMR $(100 \mathrm{MHz}$, $\left.\mathrm{CDCl}_{3}\right): \delta 24.01,27.14,28.28,29.07,40.24,41.42,41.57,42.13,42.81,44.51,46.01,46.27$, $48.23,126.16,126.62,126.66,126.85,126.91,127.28,127.66,128.39,128.95,129.13,131.47$, 132.64, 132.85, 133.70, 134.64, 135.90, 166.62, 166.64, 172.56, 172.62; Anal. Calc. for $\mathrm{C}_{23} \mathrm{H}_{27} \mathrm{~N}_{3} \mathrm{O}_{4} \mathrm{~S}$ : C, 62.56; H, 6.16; N, 9.52; found: C, 62.46; H, 6.07; N, 9.49\%; ESI-MS: $m / z$ $442.2[\mathrm{M}+\mathrm{H}]^{+}$.

N-(2-(methyl(phenyl)amino)-2-oxoethyl)-1-(phenylsulfonyl)piperidine-3-carboxamide (6k). Yield: 65\%; white solid; m.p.: 156-158 ${ }^{\circ} \mathrm{C}$; IR (KBr): 3322, 2944, 1673, 1635, 1347, $1333 \mathrm{~cm}^{-}$ ${ }^{1} ;{ }^{1} \mathrm{H}$ NMR (400 MHz, $\left.\mathrm{CDCl}_{3}\right): \delta$ 1.44-1.50 (m, 1H), 1.66-1.89 (m, 3H), 2.29-2.35 (m, 1H), 2.44-2.56 (m, 2H), 3.31 (s, 3H), 3.66-3.78 (m, 4H), 6.69 (br s, 1H), 7.20-7.23 (m, 2H), 7.38$7.48(\mathrm{~m}, 3 \mathrm{H}), 7.51-7.55(\mathrm{~m}, 2 \mathrm{H}), 7.59-7.63(\mathrm{~m}, 1 \mathrm{H}), 7.73-7.76(\mathrm{~m}, 2 \mathrm{H}) ;{ }^{13} \mathrm{C} \mathrm{NMR}(100 \mathrm{MHz}$ $\left.\mathrm{CDCl}_{3}\right): \delta 23.94,27.00,37.60,42.04,42.72,46.26,48.26,127.18,127.64,128.77,129.12$, 130.27, 132.86, 135.78, 141.68, 168.06, 172.47; Anal. Calc. for $\mathrm{C}_{21} \mathrm{H}_{25} \mathrm{~N}_{3} \mathrm{O}_{4} \mathrm{~S}: \mathrm{C}, 60.70 ; \mathrm{H}$, 6.06; N, 10.11; found: C, 60.71; H, 5.85; N, 10.12\%; ESI-MS: $m / z$ 416.1 [M+H] $]^{+}$. 


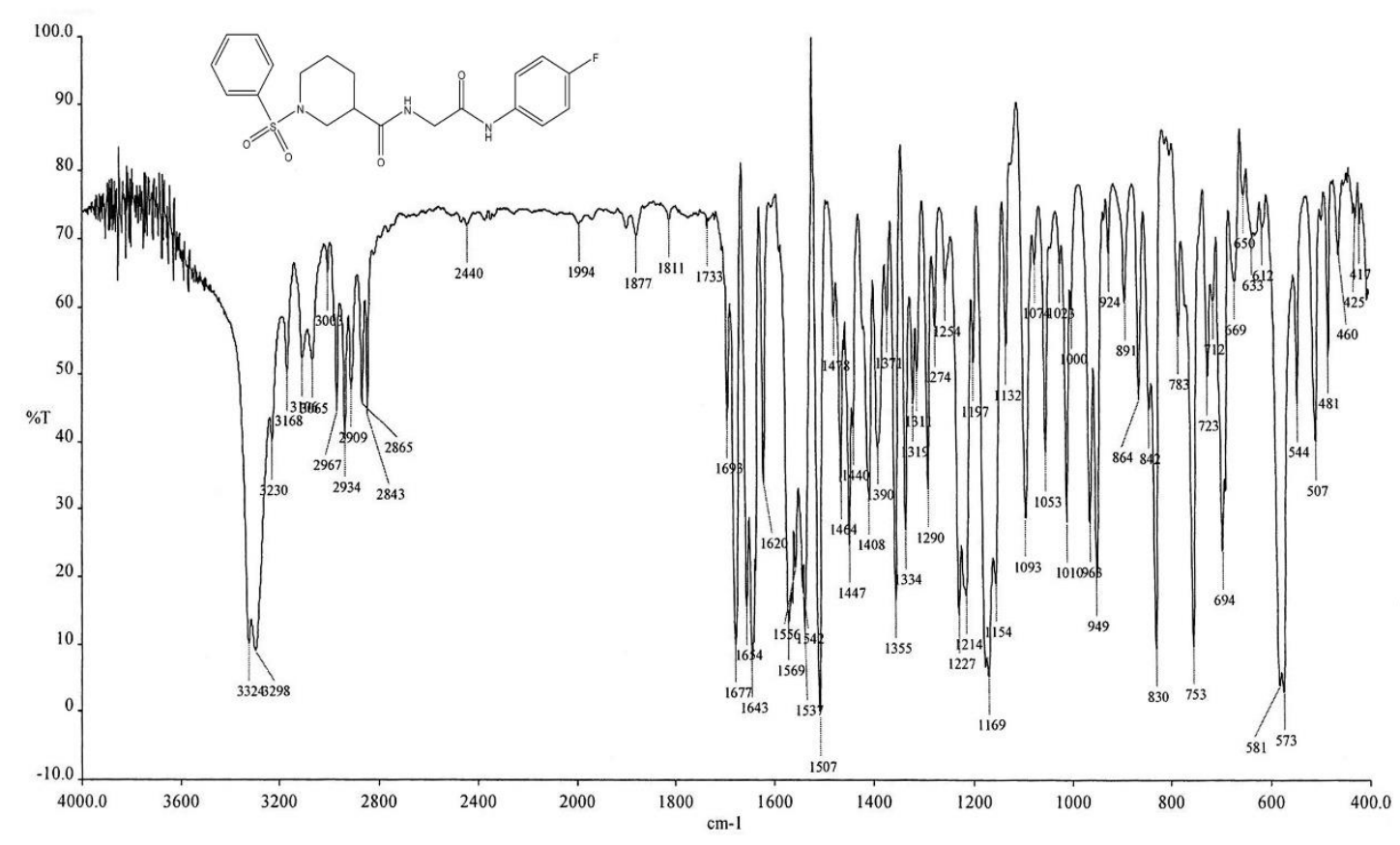

Figure 1: IR of N-(2-(4-fluorophenylamino)-2-oxoethyl)-1-(phenylsulfonyl)piperidine-3-carboxamide 6a

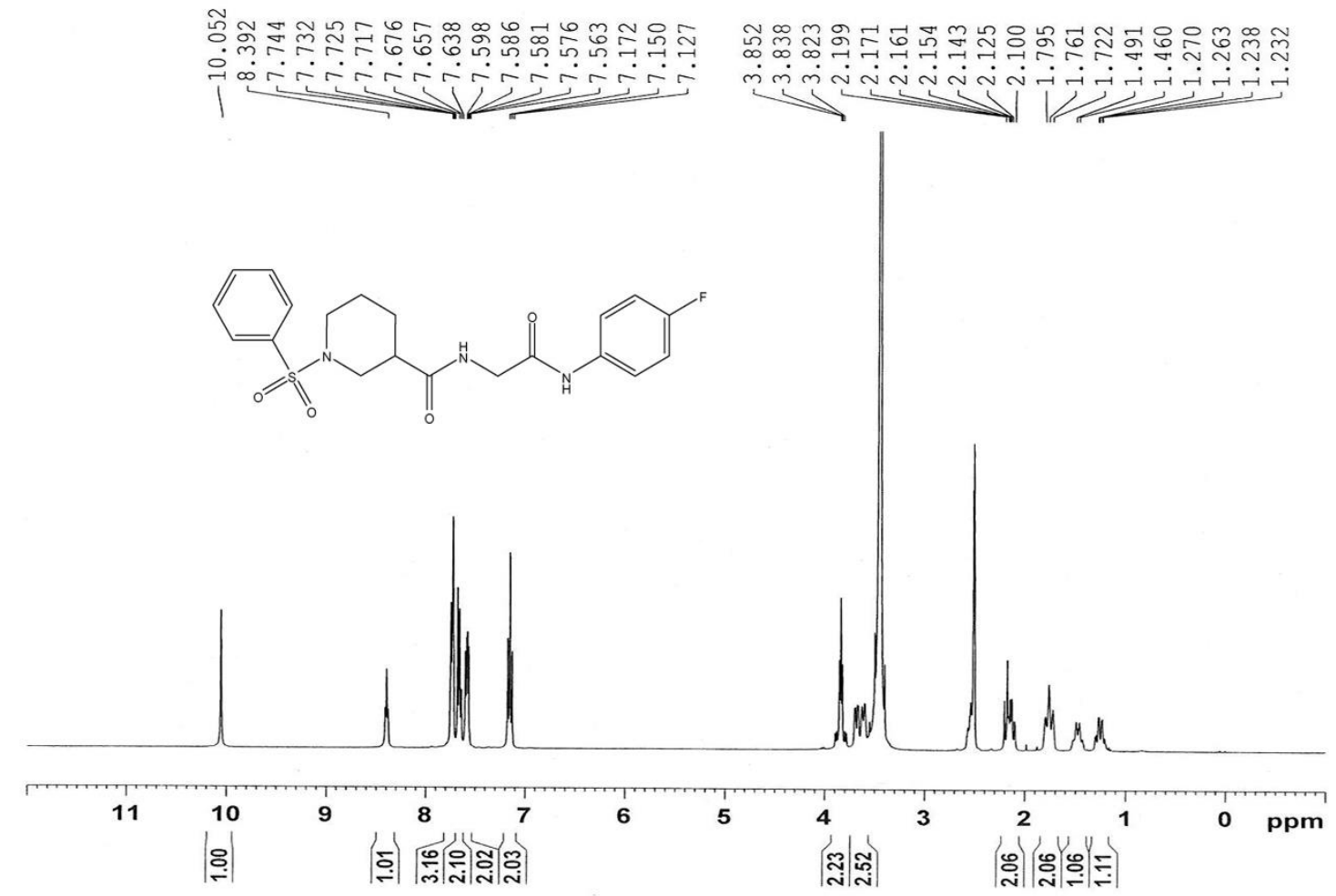

Figure 2: ${ }^{1} \mathrm{H}$ NMR of N-(2-(4-fluorophenylamino)-2-oxoethyl)-1-(phenylsulfonyl)piperidine-3-carboxamide 6a 


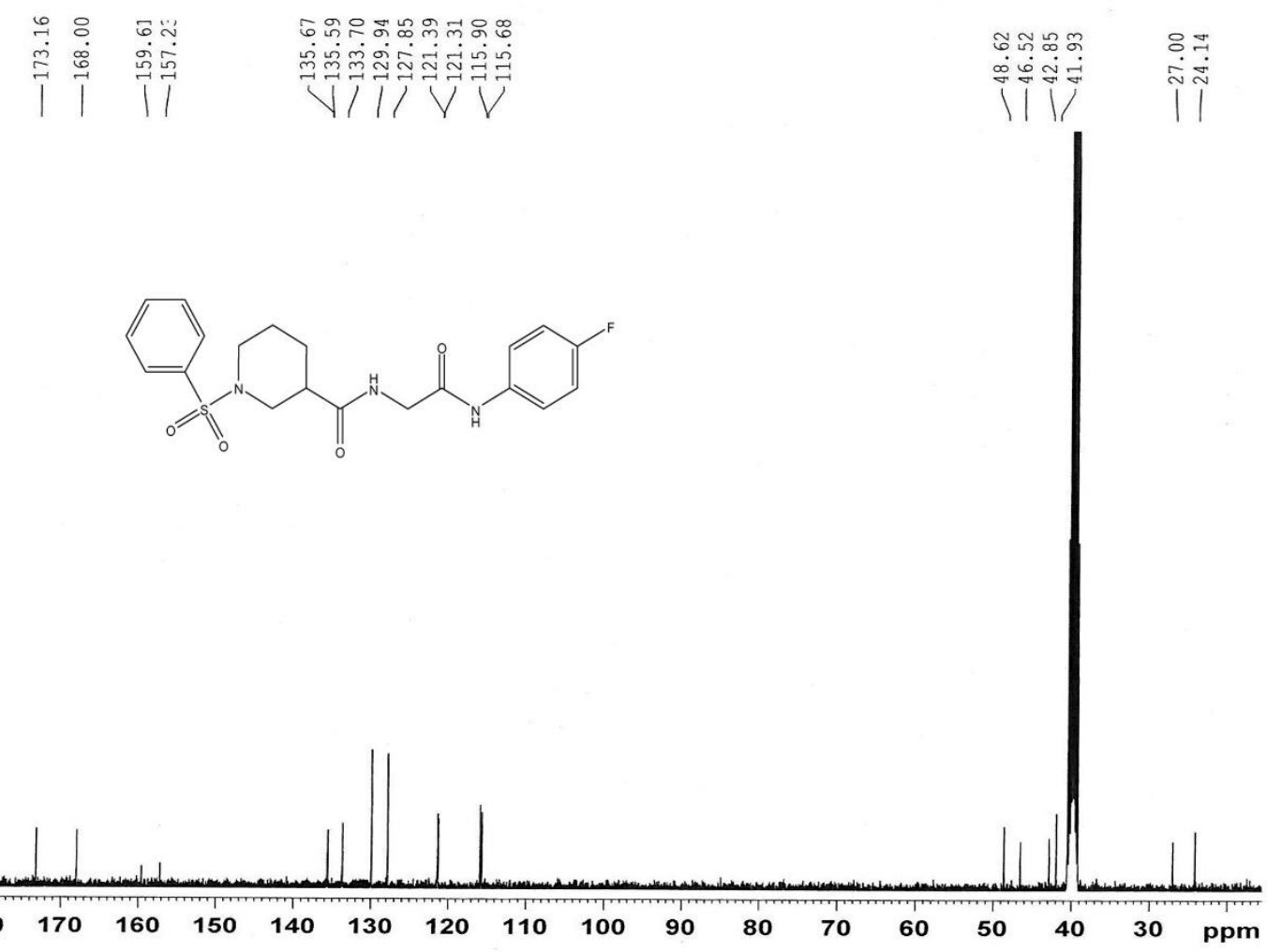

Figure 3: ${ }^{13} \mathrm{C}$ NMR of N-(2-(4-fluorophenylamino)-2-oxoethyl)-1-(phenylsulfonyl)piperidine-3-carboxamide 6a

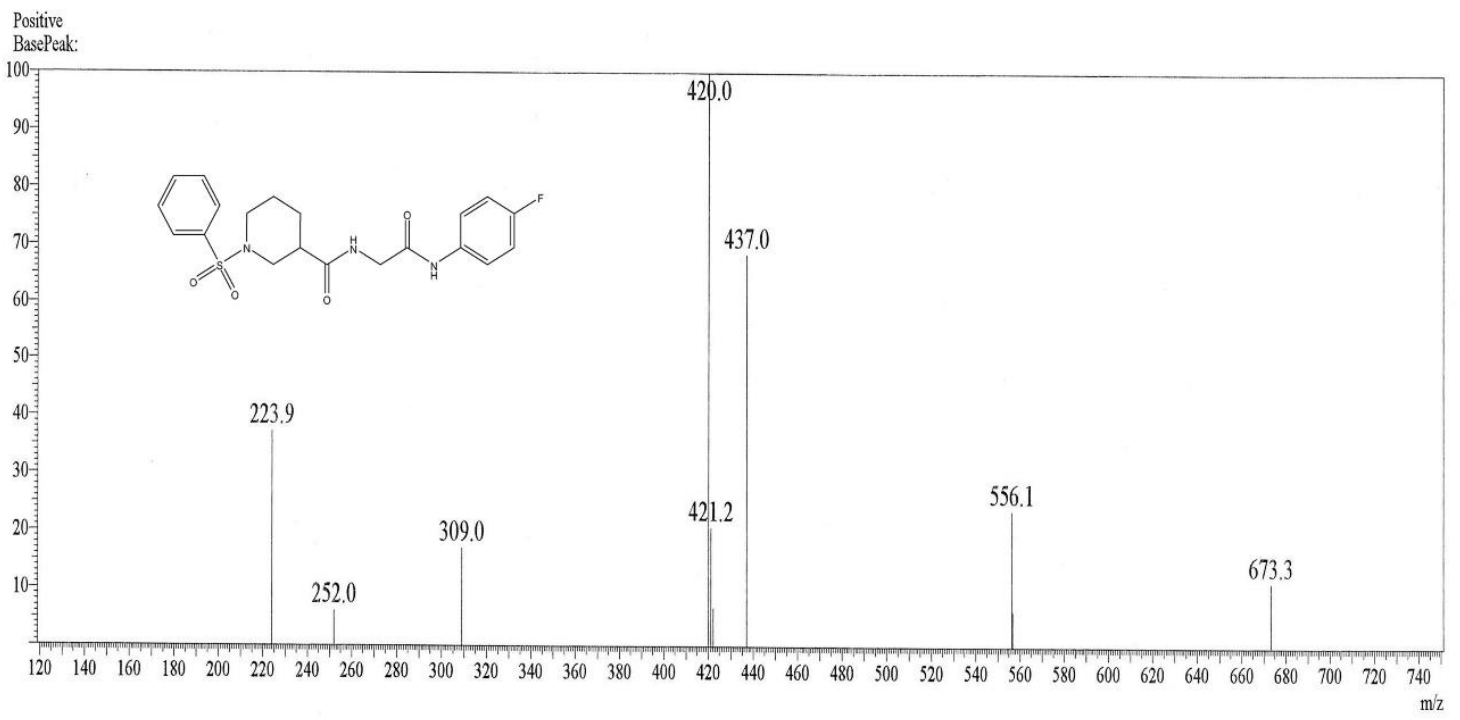

Figure 4: ESI-MS of N-(2-(4-fluorophenylamino)-2-oxoethyl)-1-(phenylsulfonyl)piperidine-3-carboxamide $6 \mathbf{a}$

9 


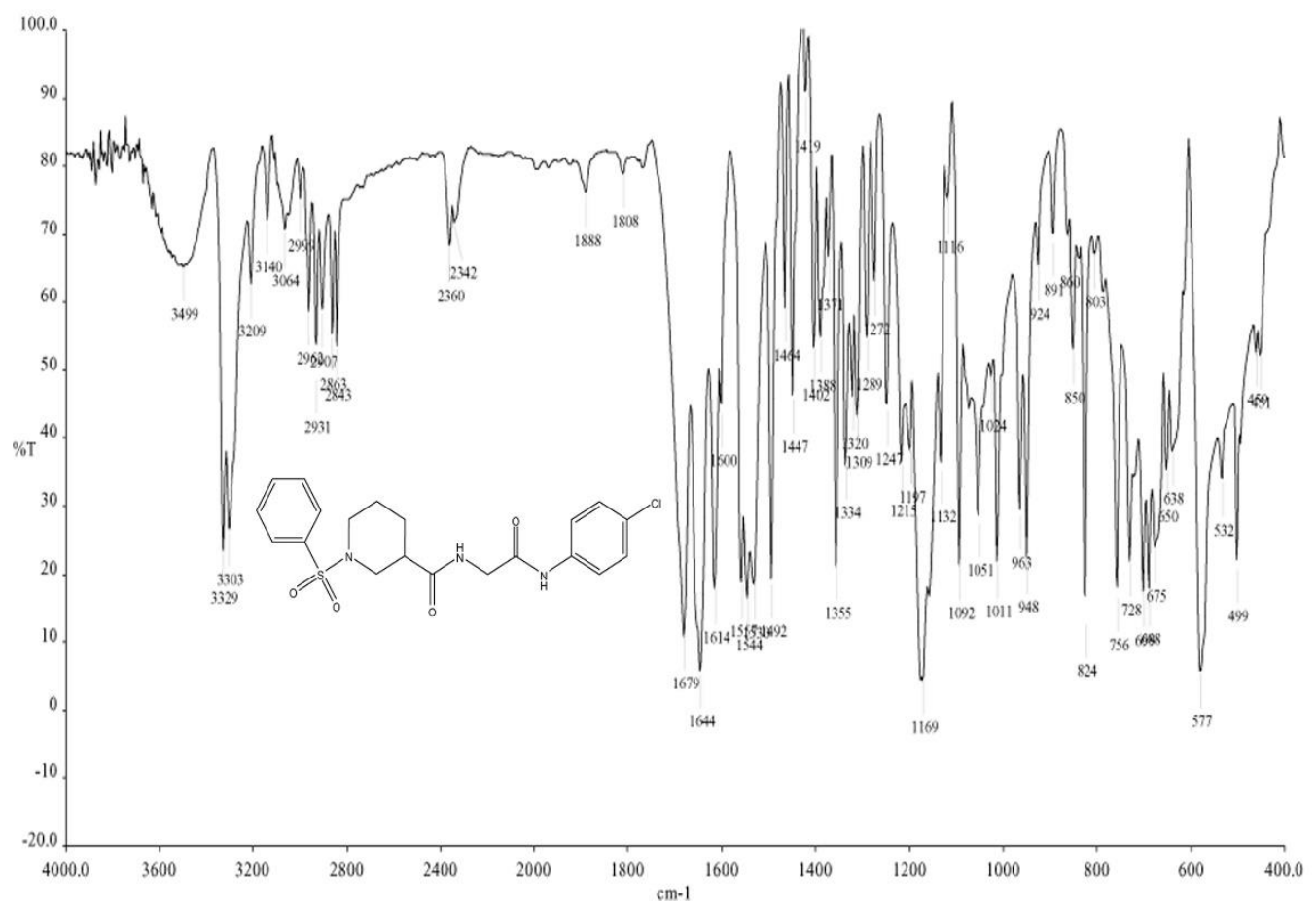

Figure 5: IR of N-(2-(4-chlorophenylamino)-2-oxoethyl)-1-(phenylsulfonyl)piperidine-3-carboxamide $\mathbf{6 b}$

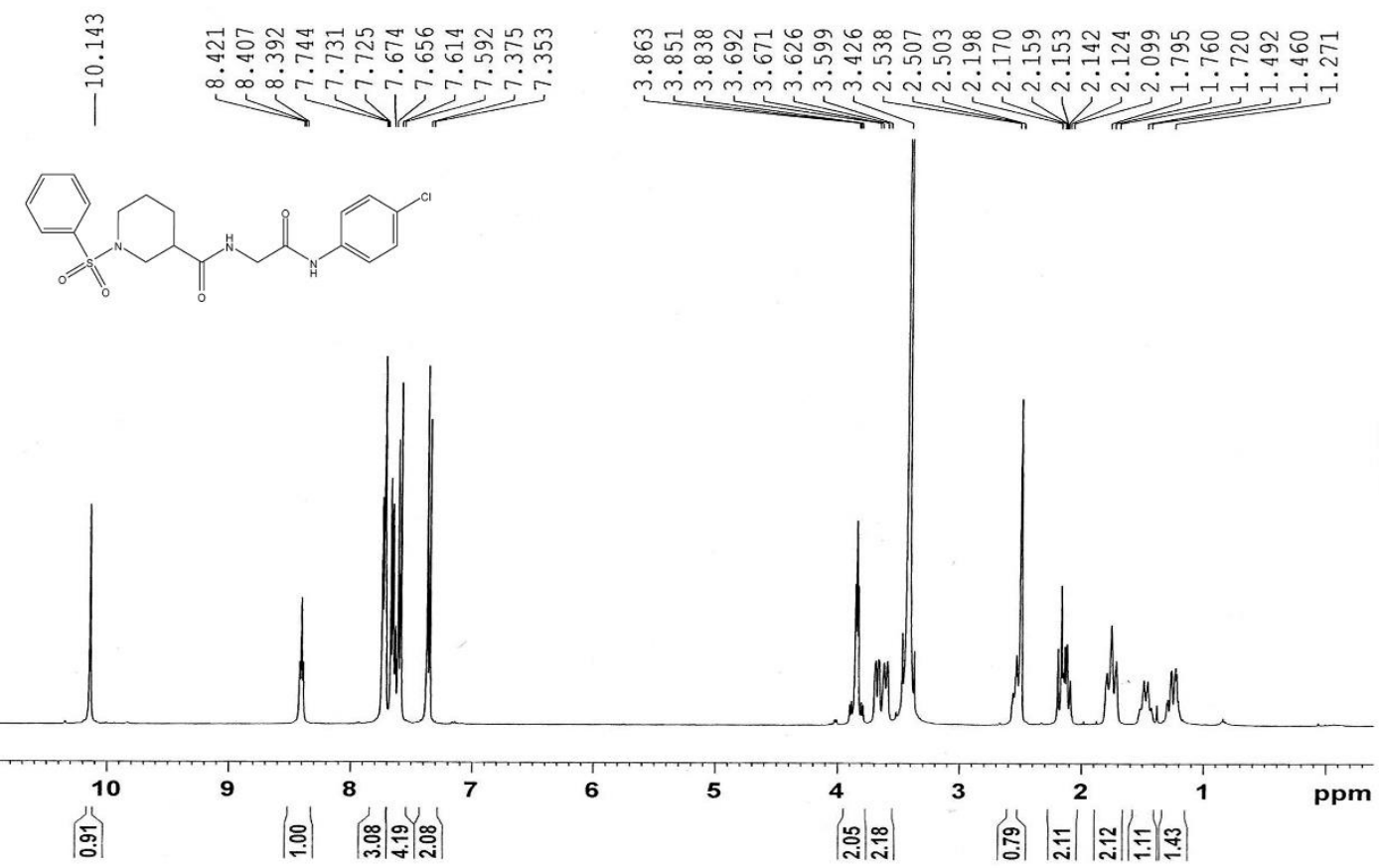

Figure 6: ${ }^{1} \mathrm{H}$ NMR of N-(2-(4-chlorophenylamino)-2-oxoethyl)-1-(phenylsulfonyl)piperidine-3-carboxamide $\mathbf{6 b}$ 

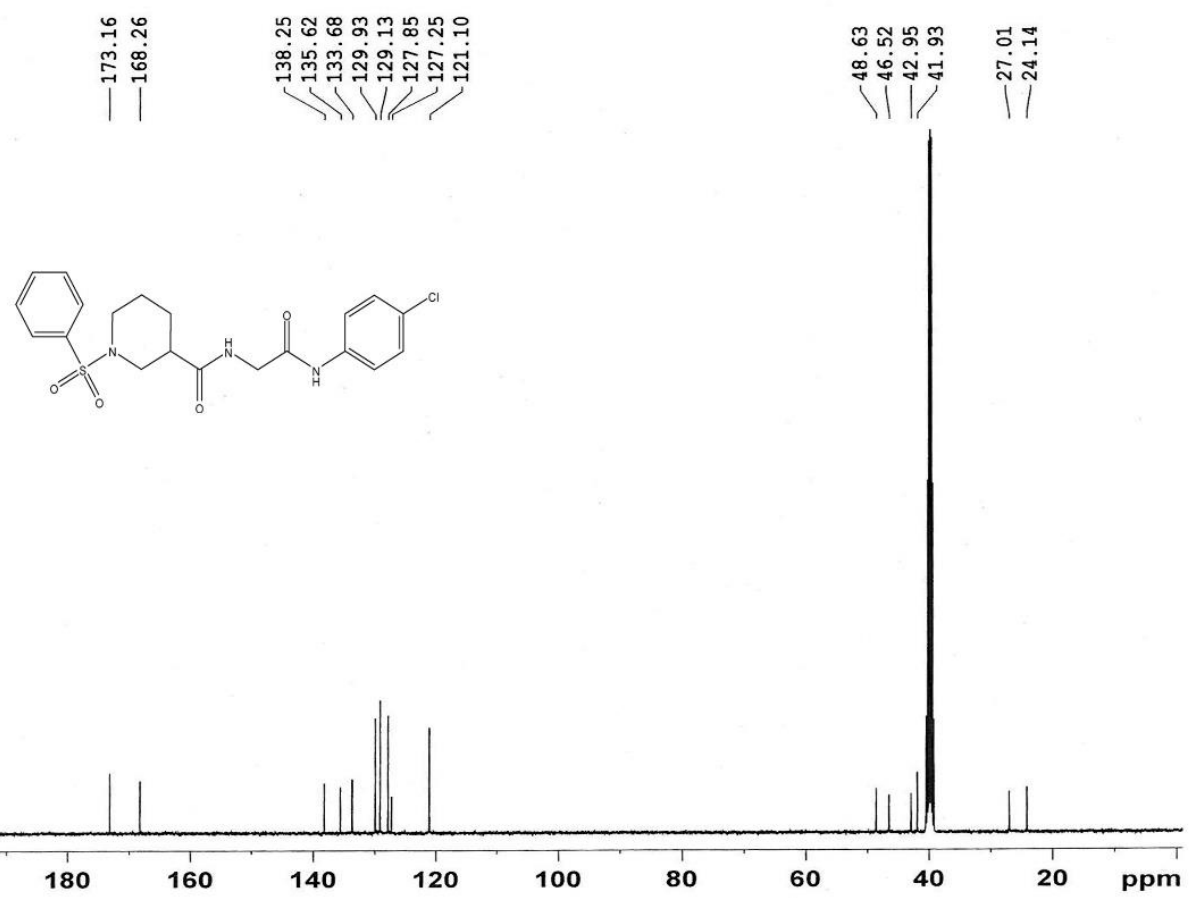

Figure 7: ${ }^{13} \mathrm{C}$ NMR of N-(2-(4-chlorophenylamino)-2-oxoethyl)-1-(phenylsulfonyl)piperidine-3carboxamide $6 \mathbf{b}$

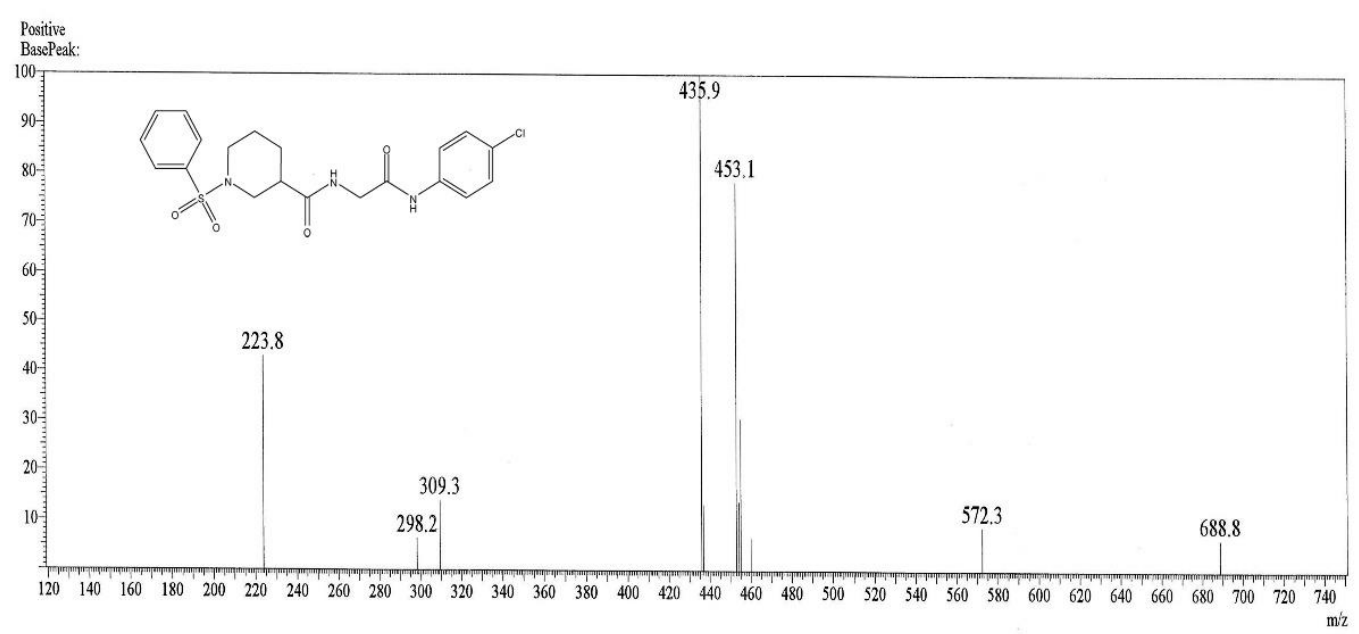

Figure 8: ESI-MS of N-(2-(4-chlorophenylamino)-2-oxoethyl)-1-(phenylsulfonyl)piperidine-3-carboxamide $\mathbf{6 b}$ 


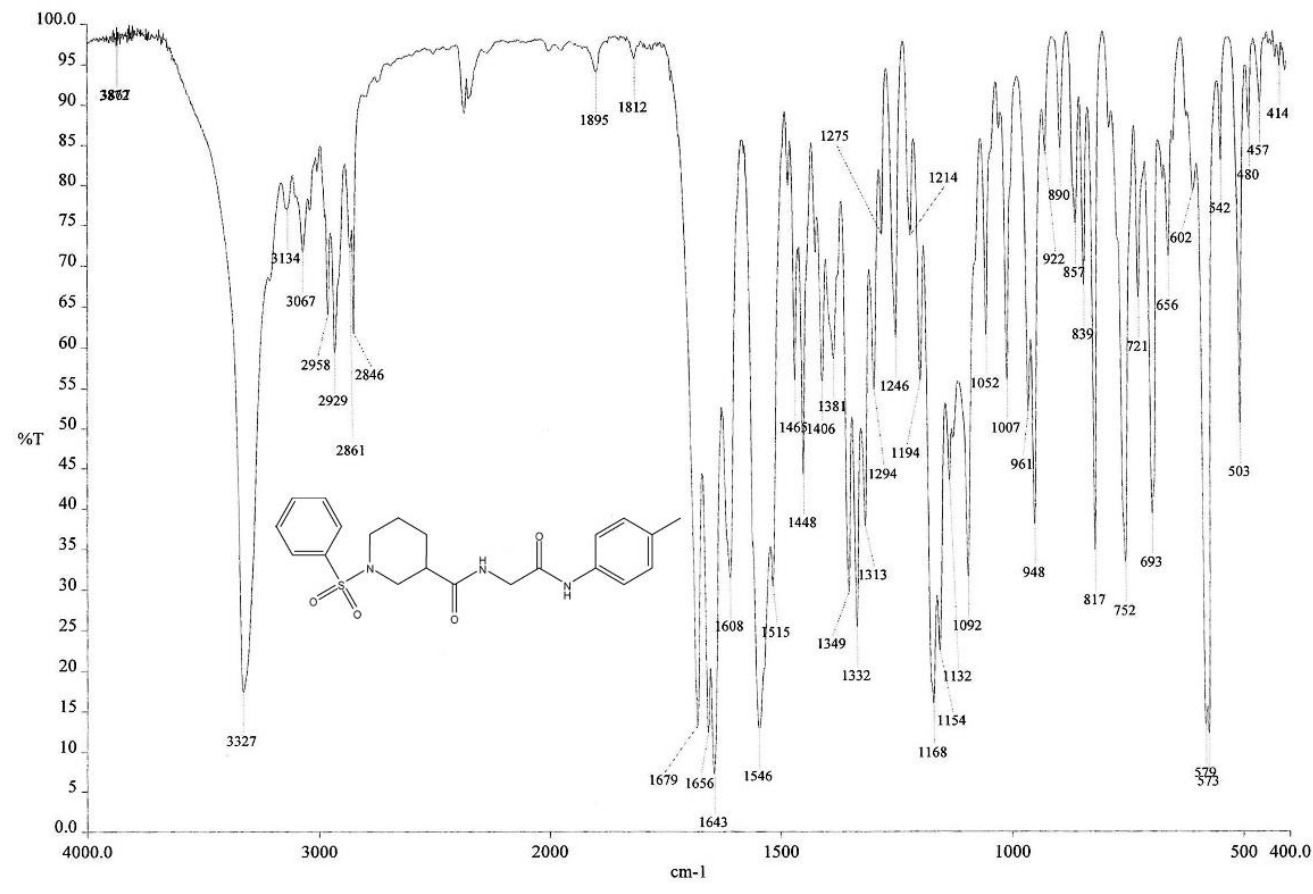

Figure 9: IR of N-(2-oxo-2-(p-tolylamino)ethyl)-1-(phenylsulfonyl)piperidine-3-carboxamide $6 \mathbf{c}$

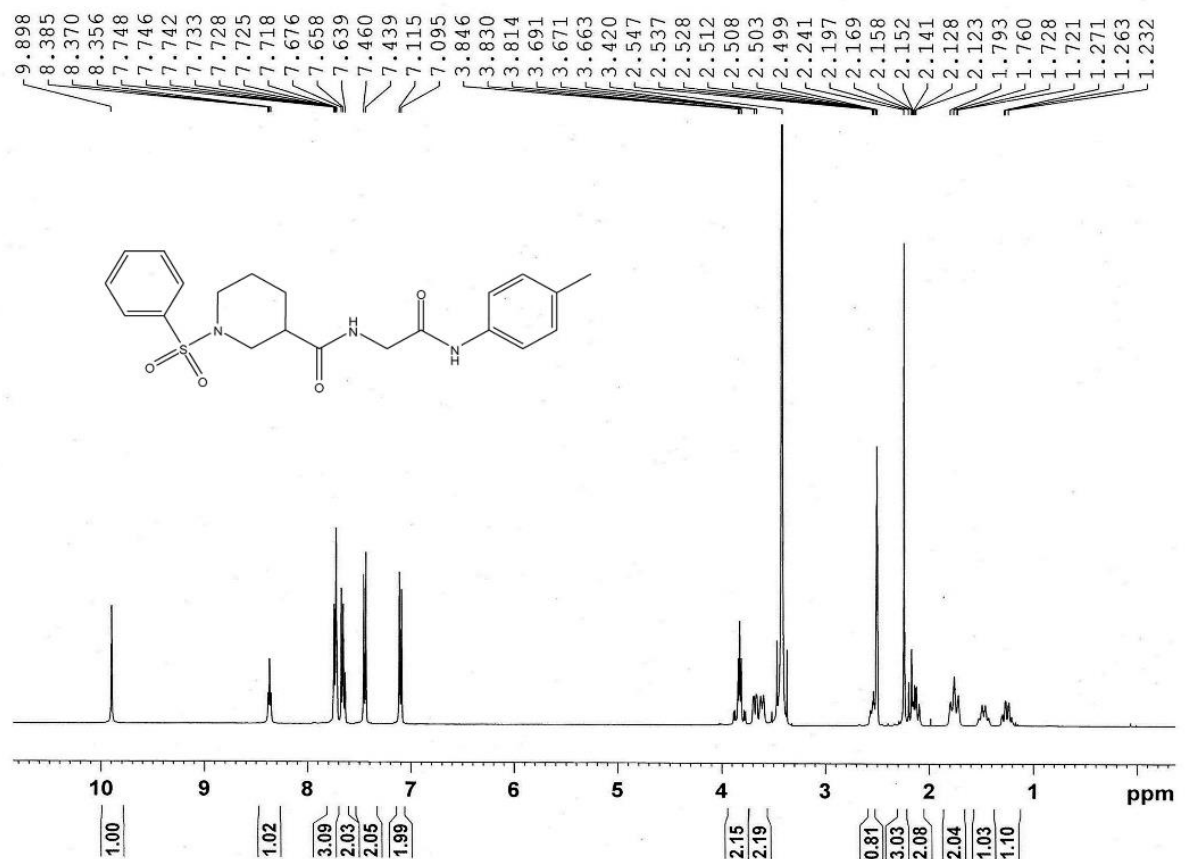

Figure 10: ${ }^{1} \mathrm{H}$ NMR of N-(2-oxo-2-(p-tolylamino)ethyl)-1-(phenylsulfonyl)piperidine-3-carboxamide $\mathbf{6 c}$ 


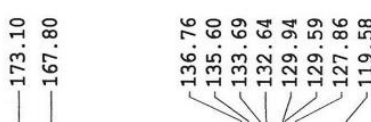

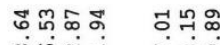

هั
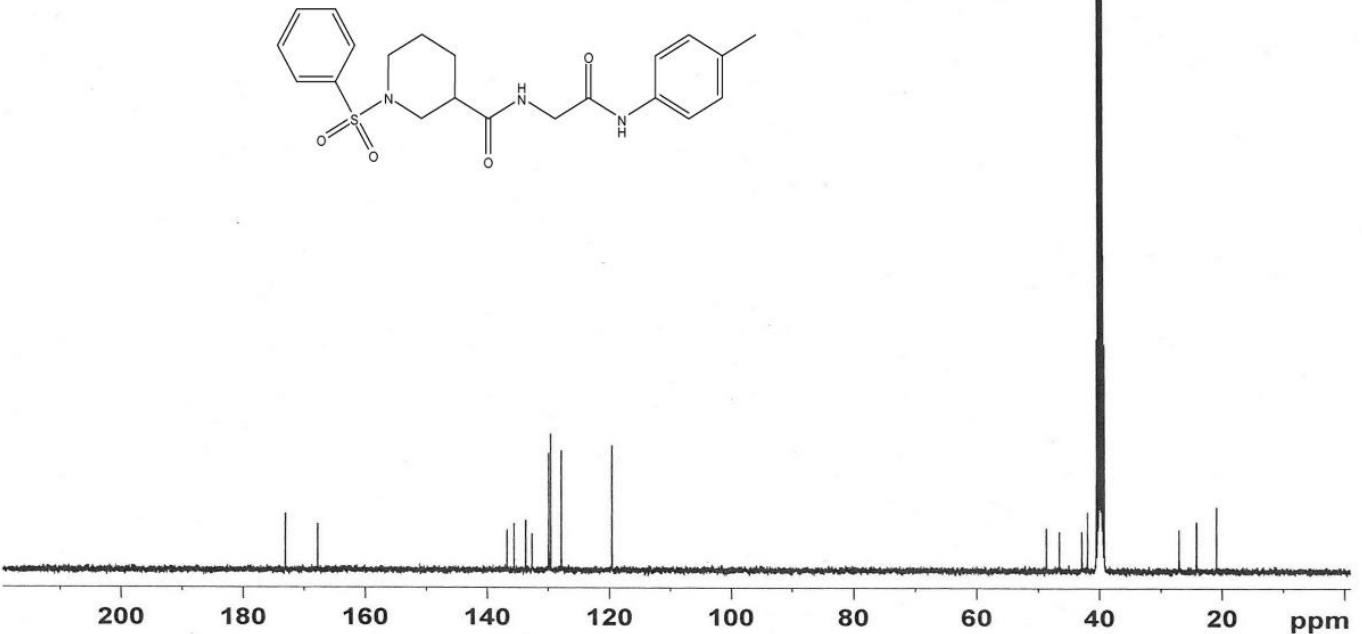

Figure 11: ${ }^{13} \mathrm{C}$ NMR of N-(2-oxo-2-(p-tolylamino)ethyl)-1-(phenylsulfonyl)piperidine-3-carboxamide $\mathbf{6 c}$

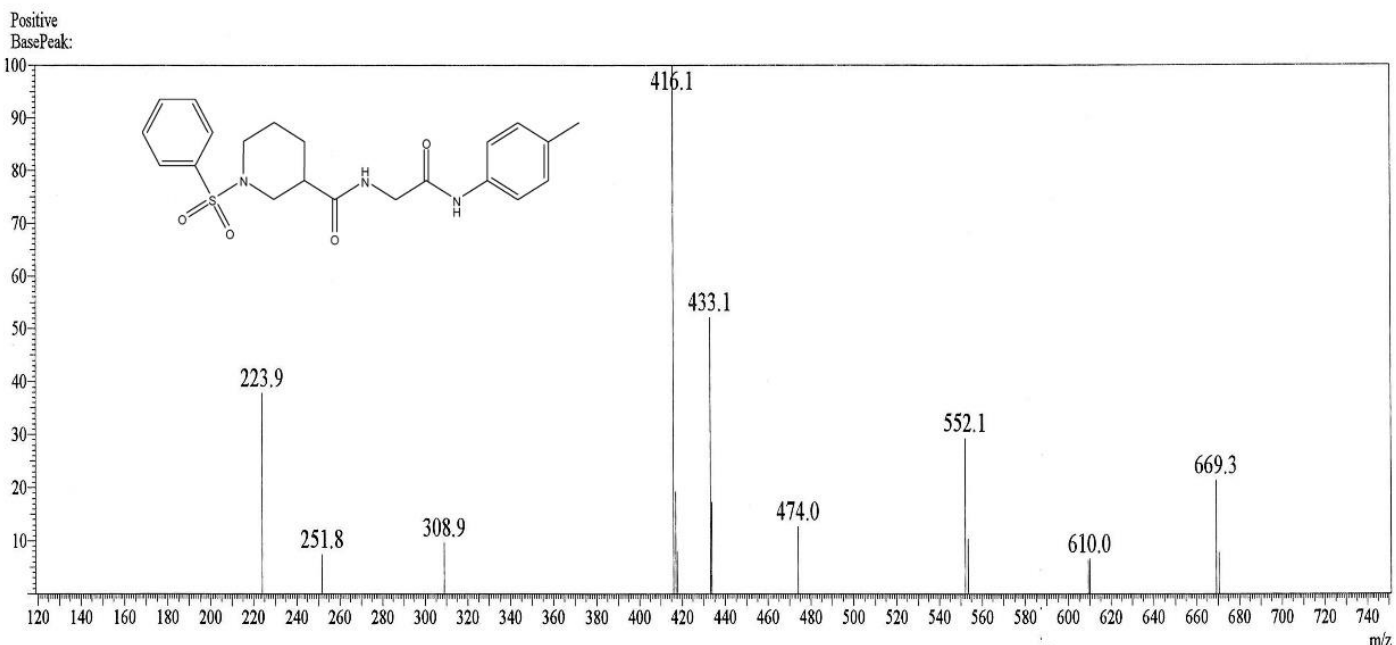

Figure 12: ESI-MS of N-(2-oxo-2-(p-tolylamino)ethyl)-1-(phenylsulfonyl)piperidine-3-carboxamide $\mathbf{6 c}$ 


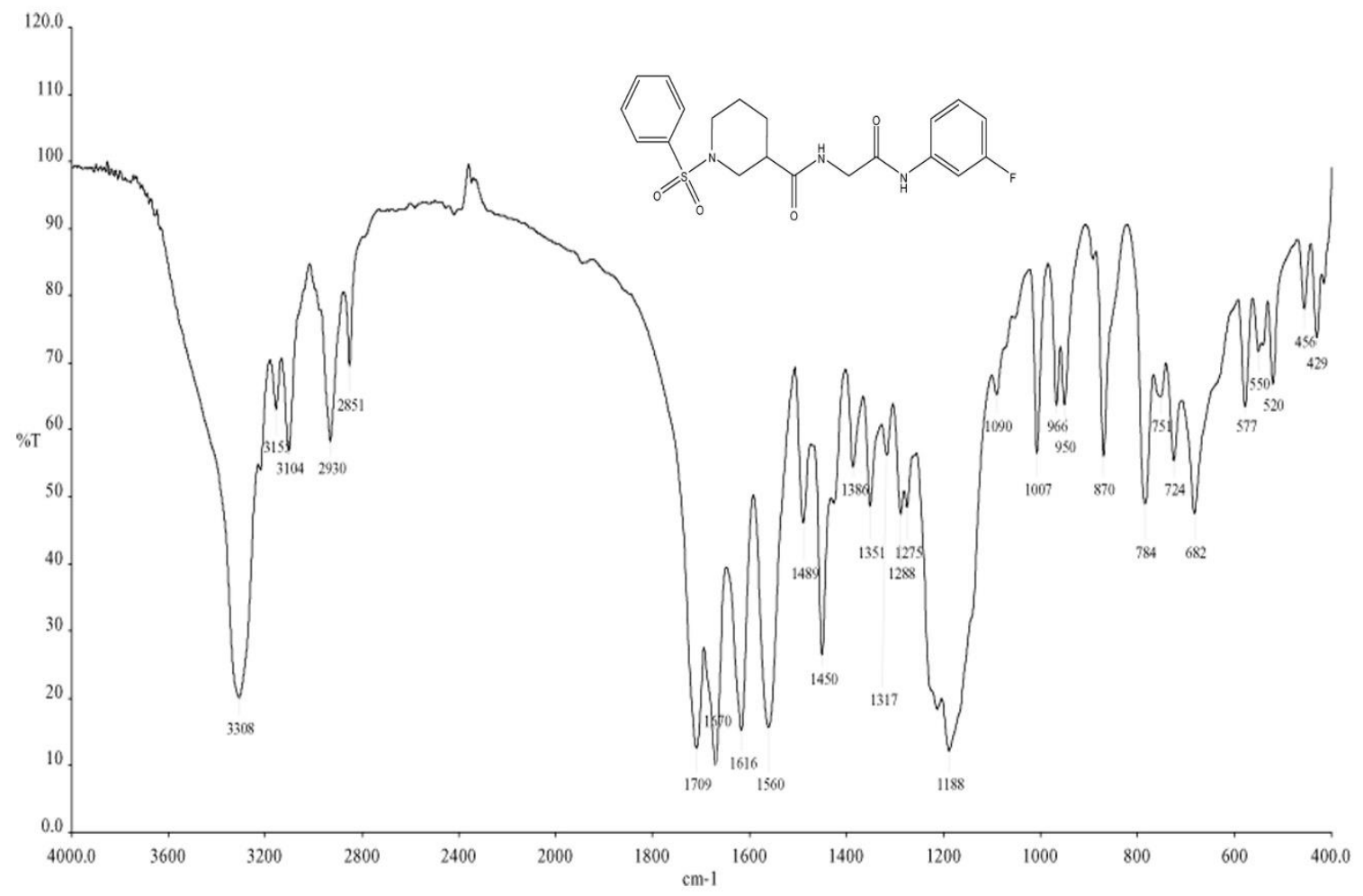

Figure 13: IR of N-(2-(3-fluorophenylamino)-2-oxoethyl)-1-(phenylsulfonyl)piperidine-3-carboxamide $6 \mathbf{d}$

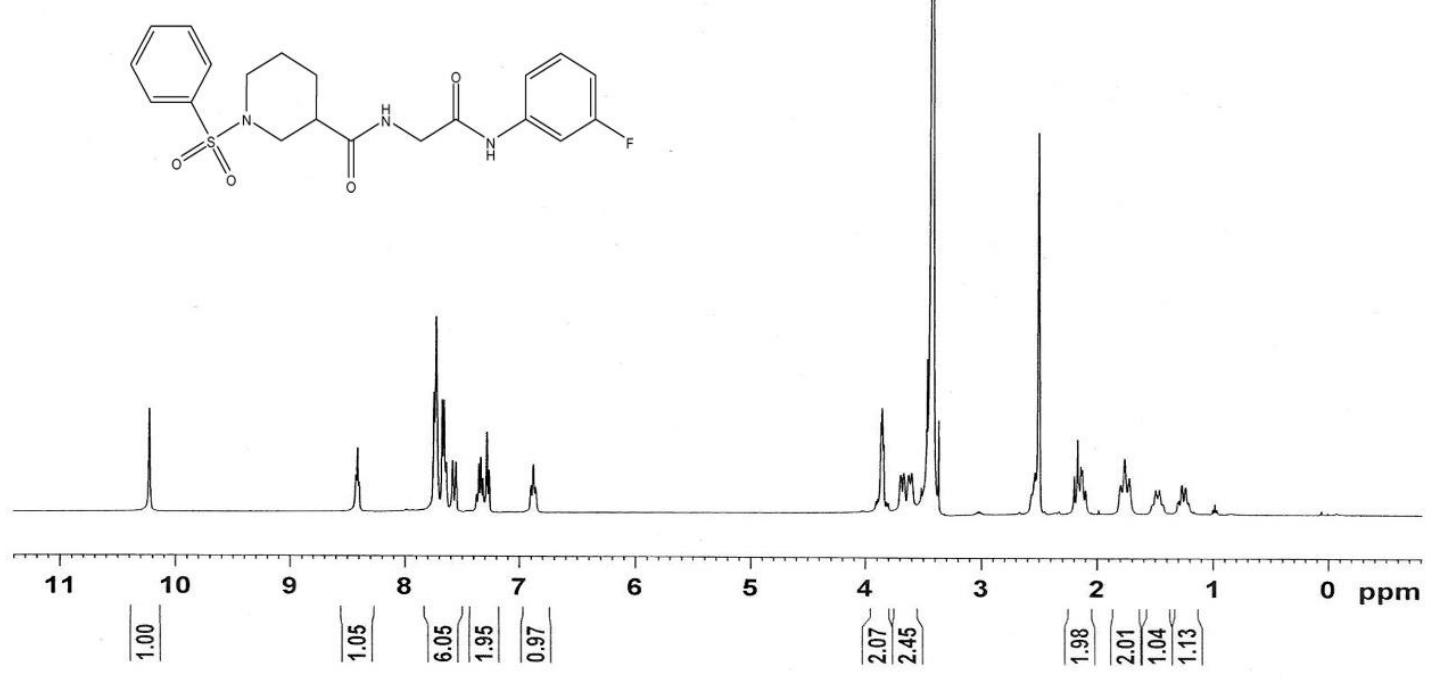

14 
Figure 14: ${ }^{1} \mathrm{H}$ NMR of N-(2-(3-fluorophenylamino)-2-oxoethyl)-1-(phenylsulfonyl)piperidine-3-carboxamide

\section{6d}

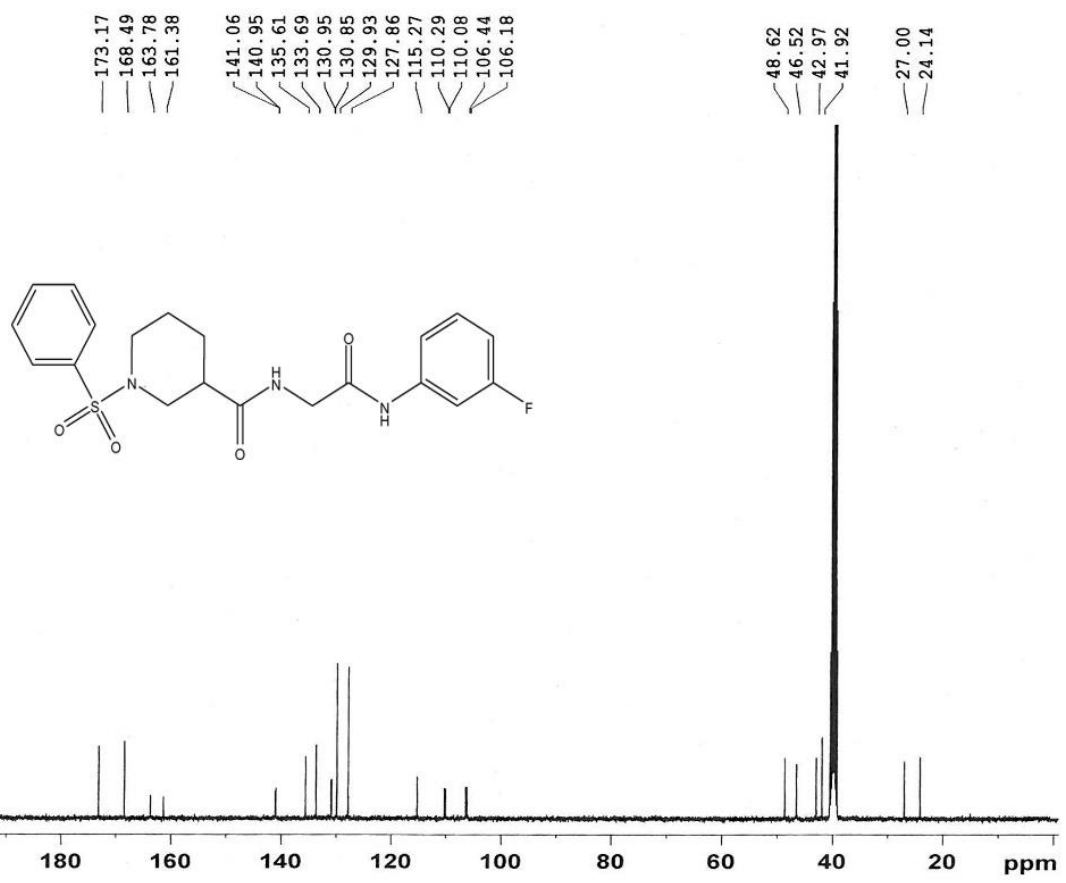

Figure 15: ${ }^{13} \mathrm{C}$ NMR of N-(2-(3-fluorophenylamino)-2-oxoethyl)-1-(phenylsulfonyl)piperidine-3-carboxamide 6d

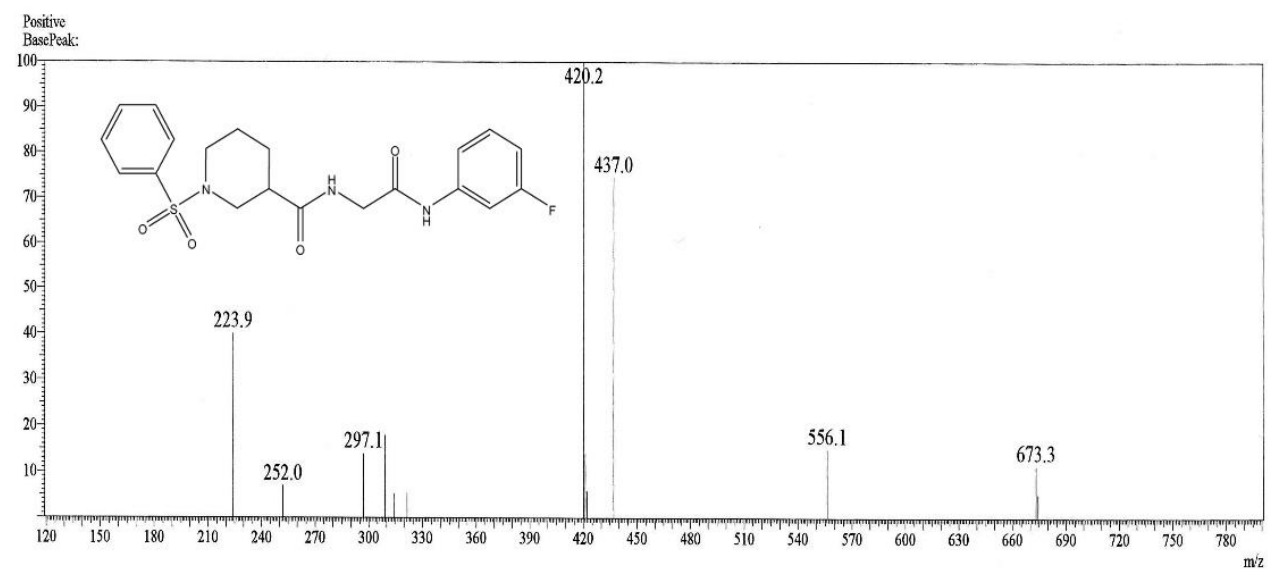


Figure 16: ESI-MS of N-(2-(3-fluorophenylamino)-2-oxoethyl)-1-(phenylsulfonyl)piperidine-3-carboxamide 6d

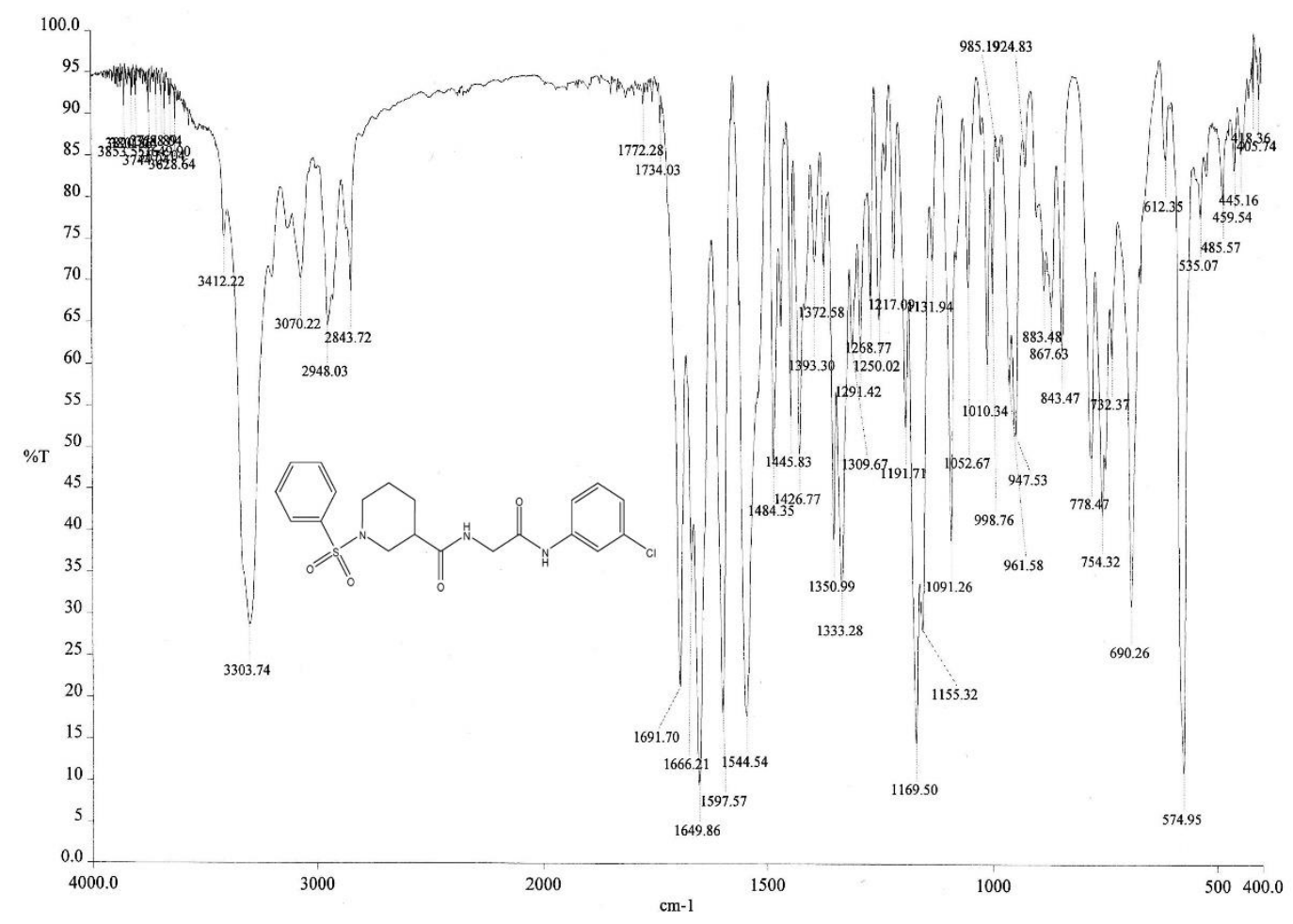

Figure 17: IR of N-(2-(3-chlorophenylamino)-2-oxoethyl)-1-(phenylsulfonyl)piperidine-3-carboxamide 6e

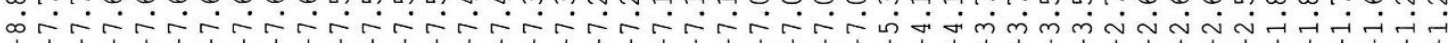<smiles>O=C(CNC(=O)C1CCCN(S(=O)(=O)c2ccccc2)C1)Nc1cccc(Cl)c1</smiles>

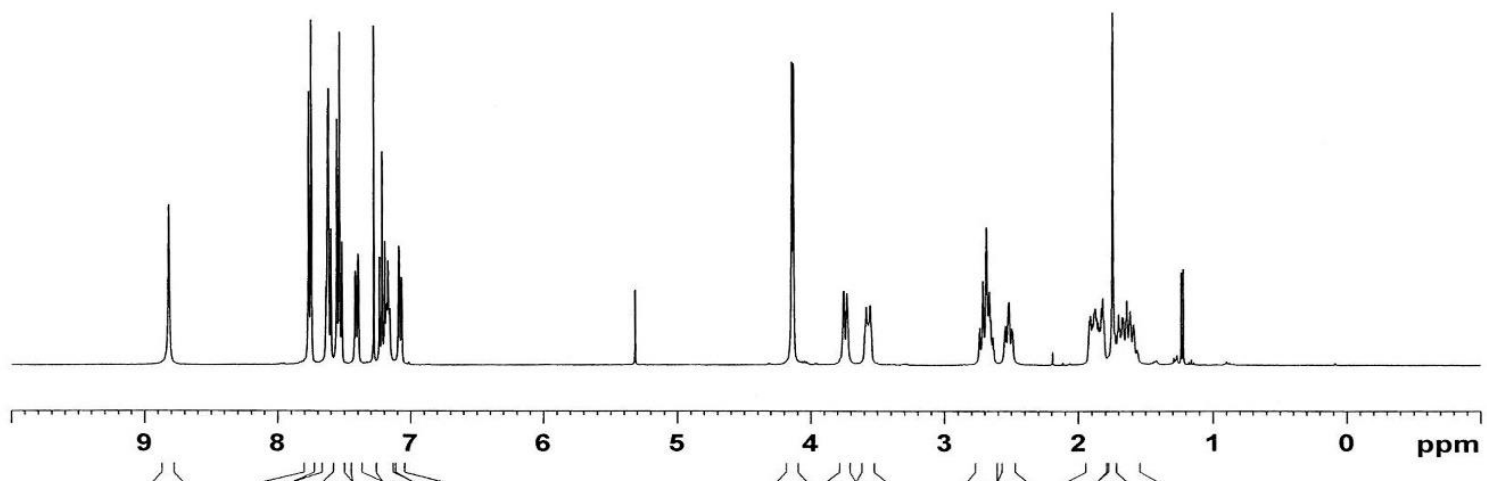

( 
Figure 18: ${ }^{1} \mathrm{H}$ NMR of N-(2-(3-chlorophenylamino)-2-oxoethyl)-1-(phenylsulfonyl)piperidine-3-carboxamide $6 e$

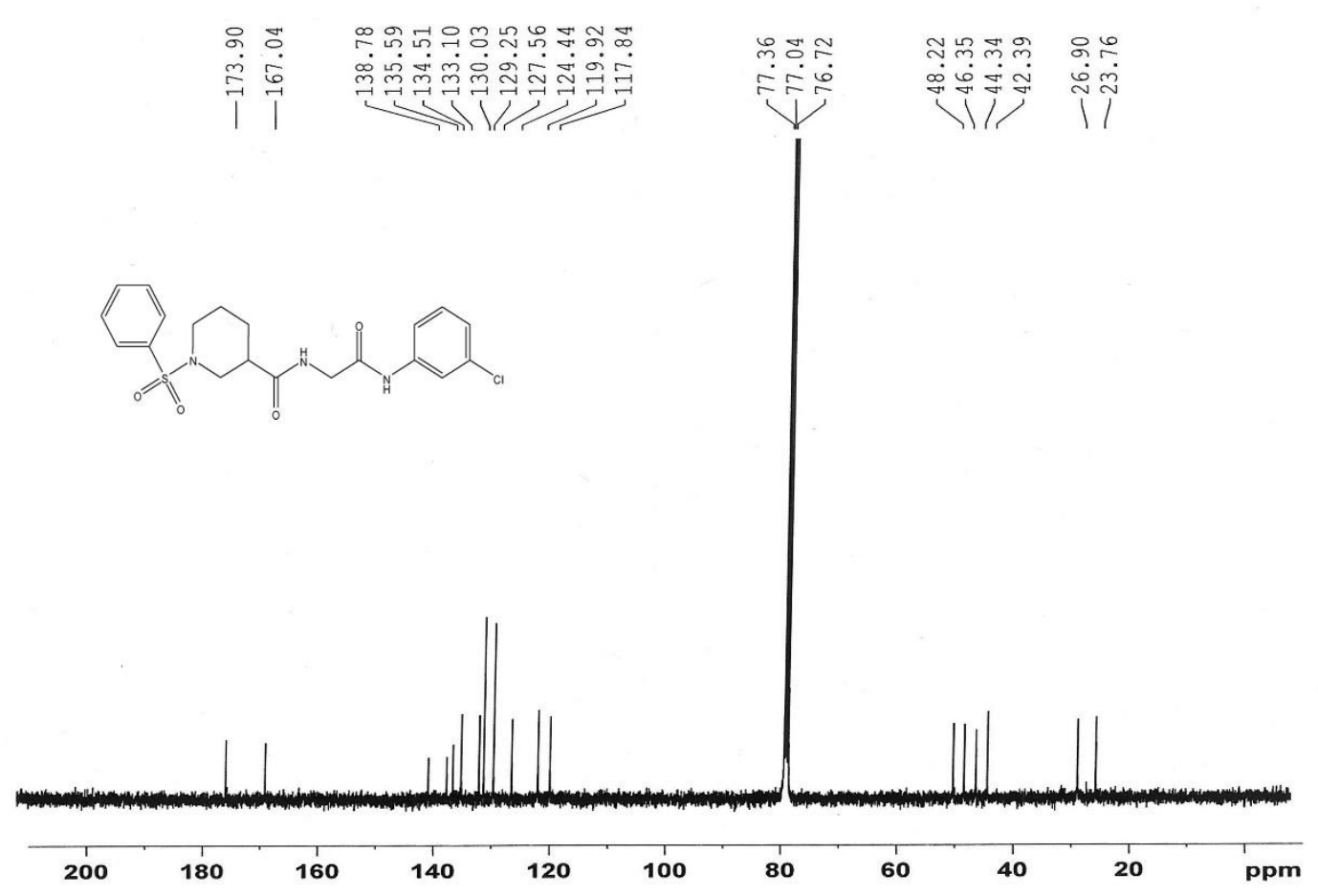

Figure 19: ${ }^{13} \mathrm{C}$ NMR of N-(2-(3-chlorophenylamino)-2-oxoethyl)-1-(phenylsulfonyl)piperidine-3-carboxamide 6e

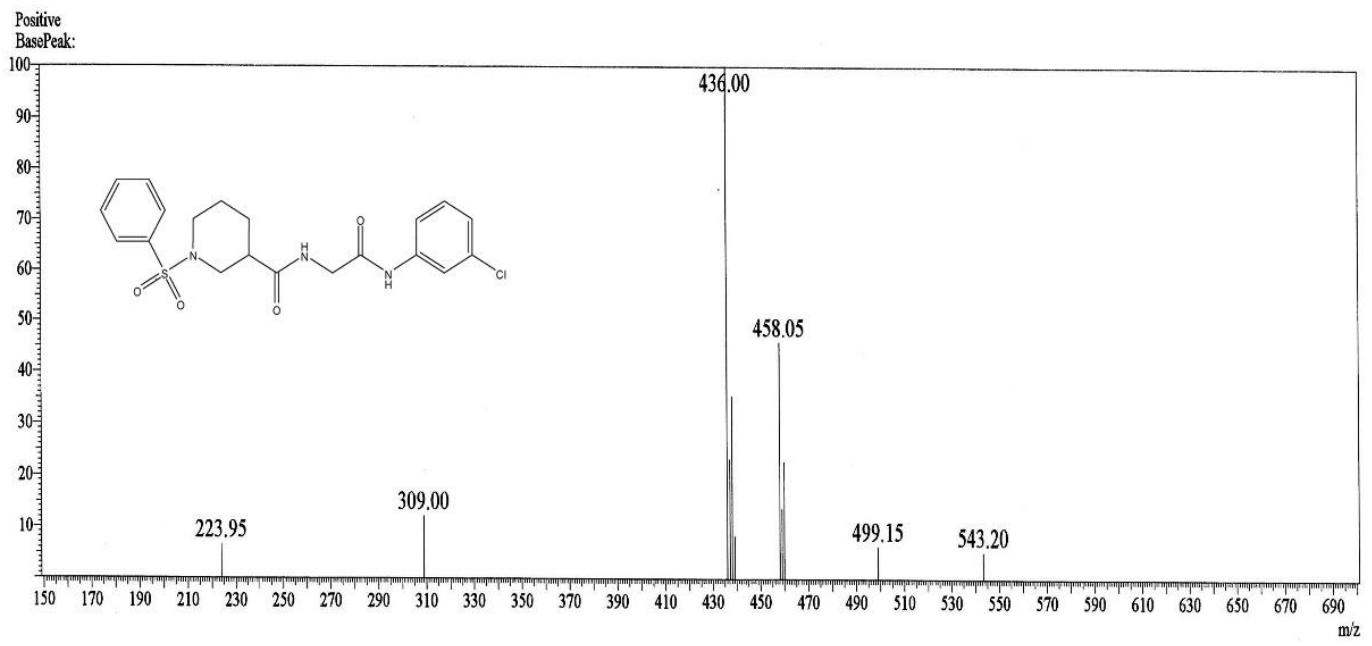

17 
Figure 20: ESI-MS of N-(2-(3-chlorophenylamino)-2-oxoethyl)-1-(phenylsulfonyl)piperidine-3-carboxamide $6 \mathbf{e}$

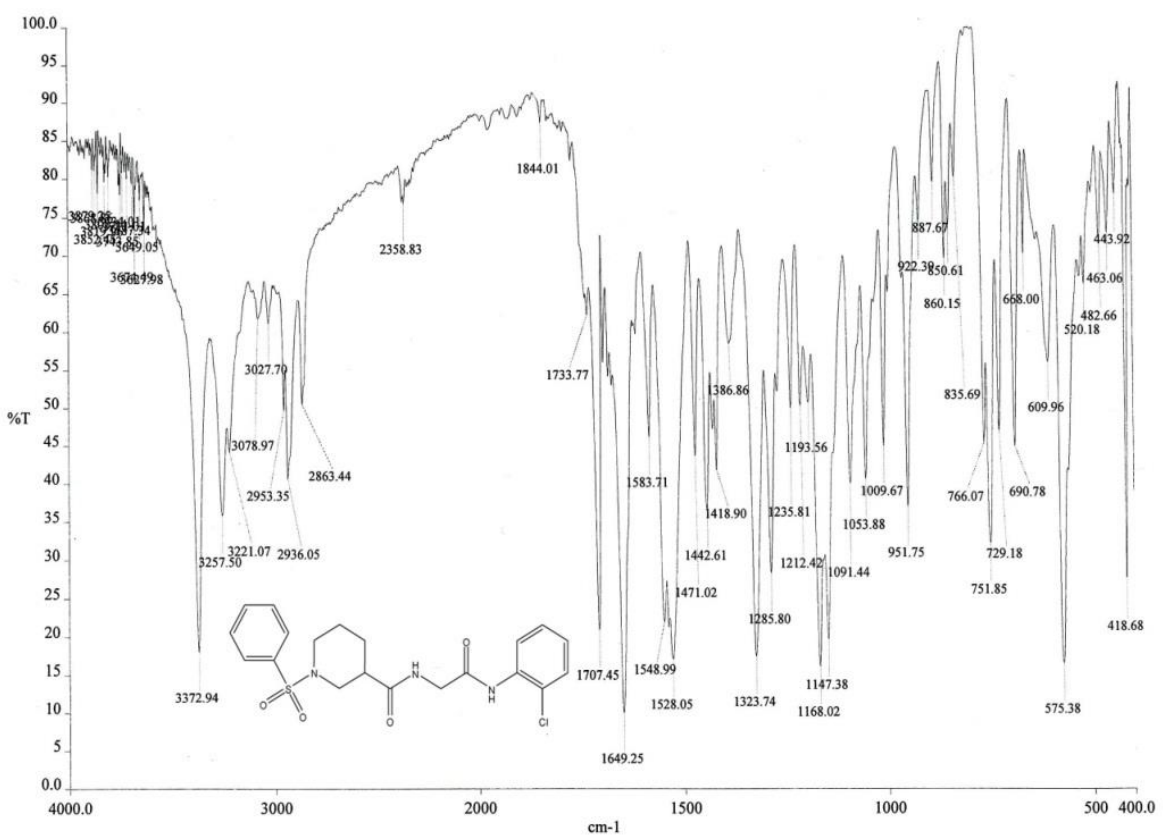

Figure 21: IR of N-(2-(2-chlorophenylamino)-2-oxoethyl)-1-(phenylsulfonyl)piperidine-3-carboxamide $6 \mathbf{f}$

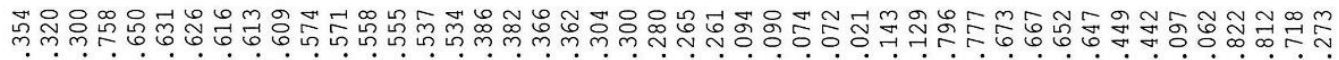 iriri}
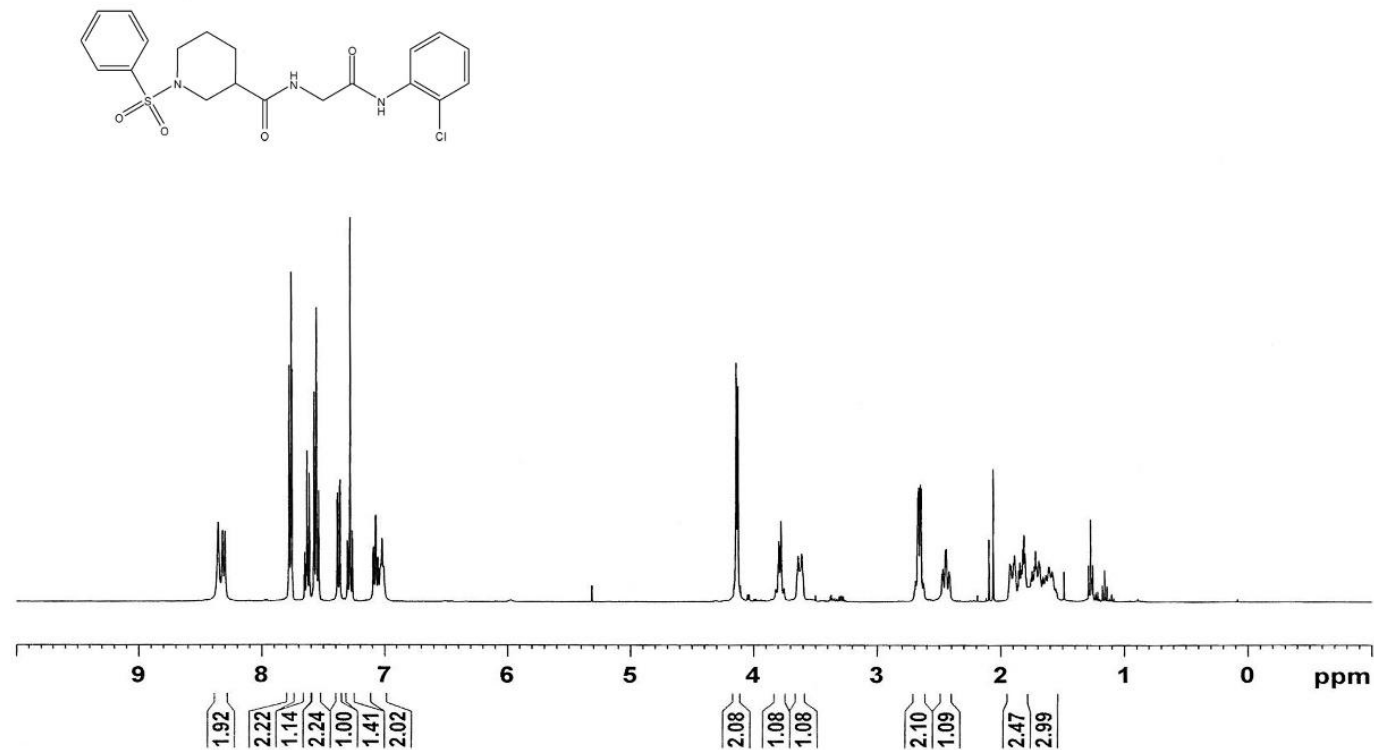

Figure 22: ${ }^{1} \mathrm{H}$ NMR of N-(2-(2-chlorophenylamino)-2-oxoethyl)-1-(phenylsulfonyl)piperidine-3-carboxamide $6 \mathbf{f}$ 


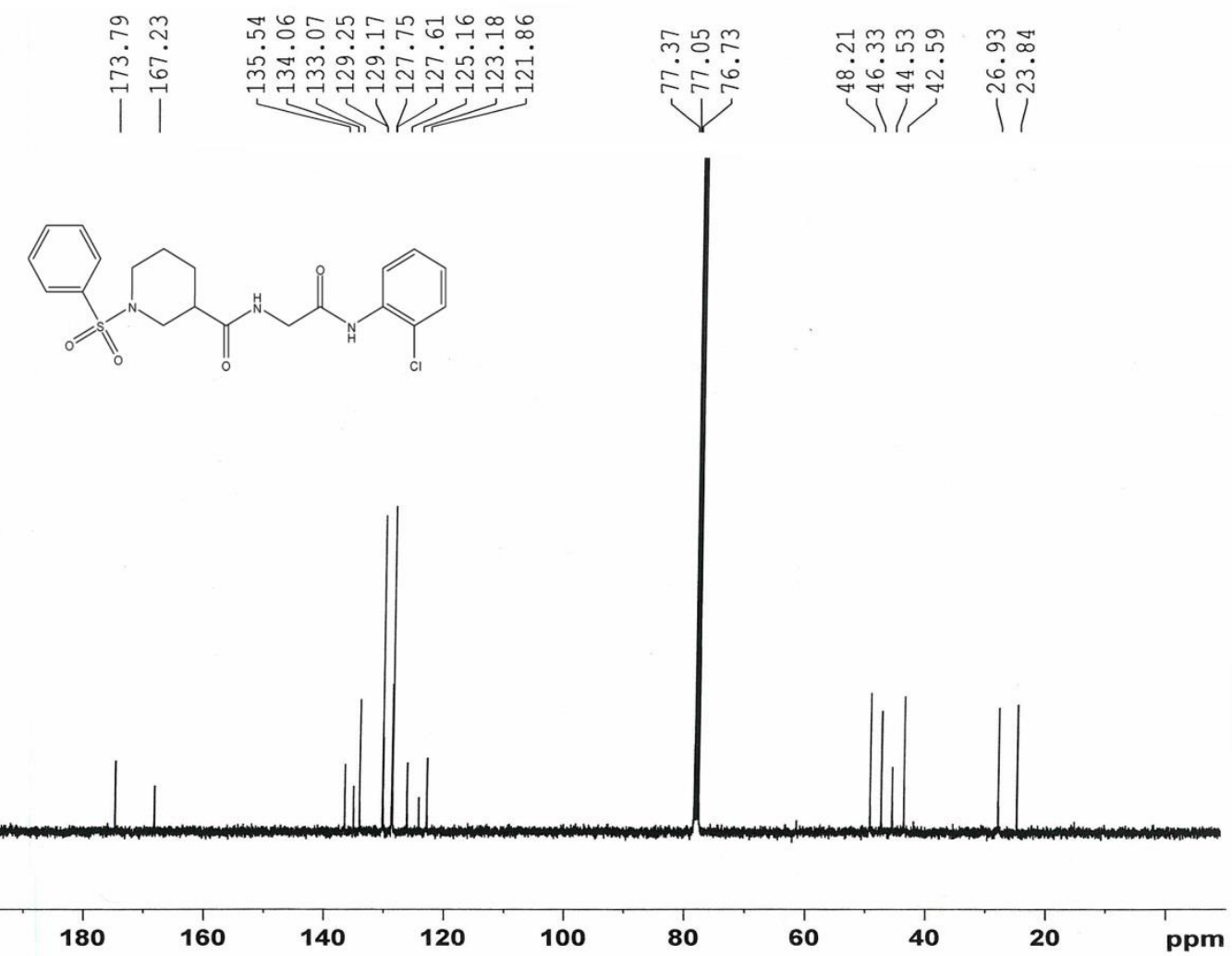

Figure $23:{ }^{13} \mathrm{C}$ NMR of N-(2-(2-chlorophenylamino)-2-oxoethyl)-1-(phenylsulfonyl)piperidine-3-carboxamide 6f

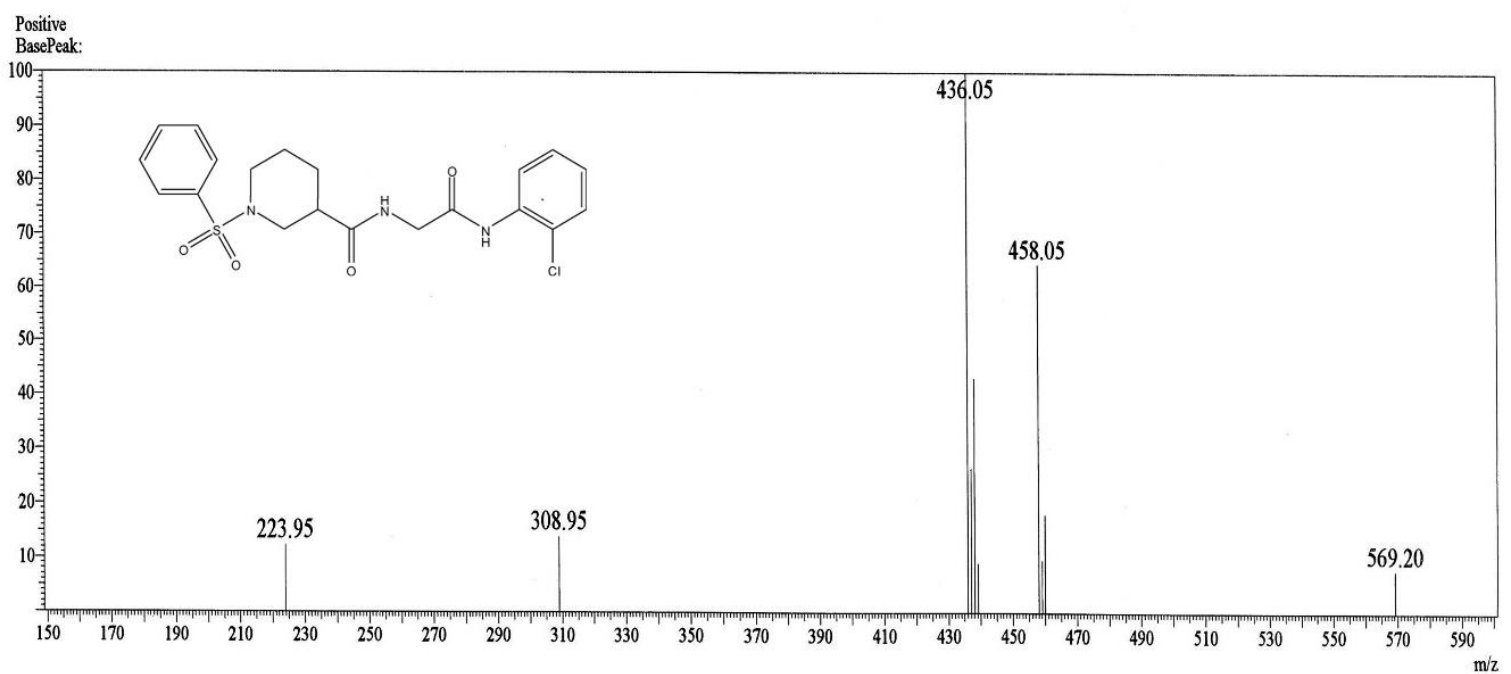

Figure 24: ESI-MS of N-(2-(2-chlorophenylamino)-2-oxoethyl)-1-(phenylsulfonyl)piperidine-3-carboxamide $6 \mathbf{f}$ 


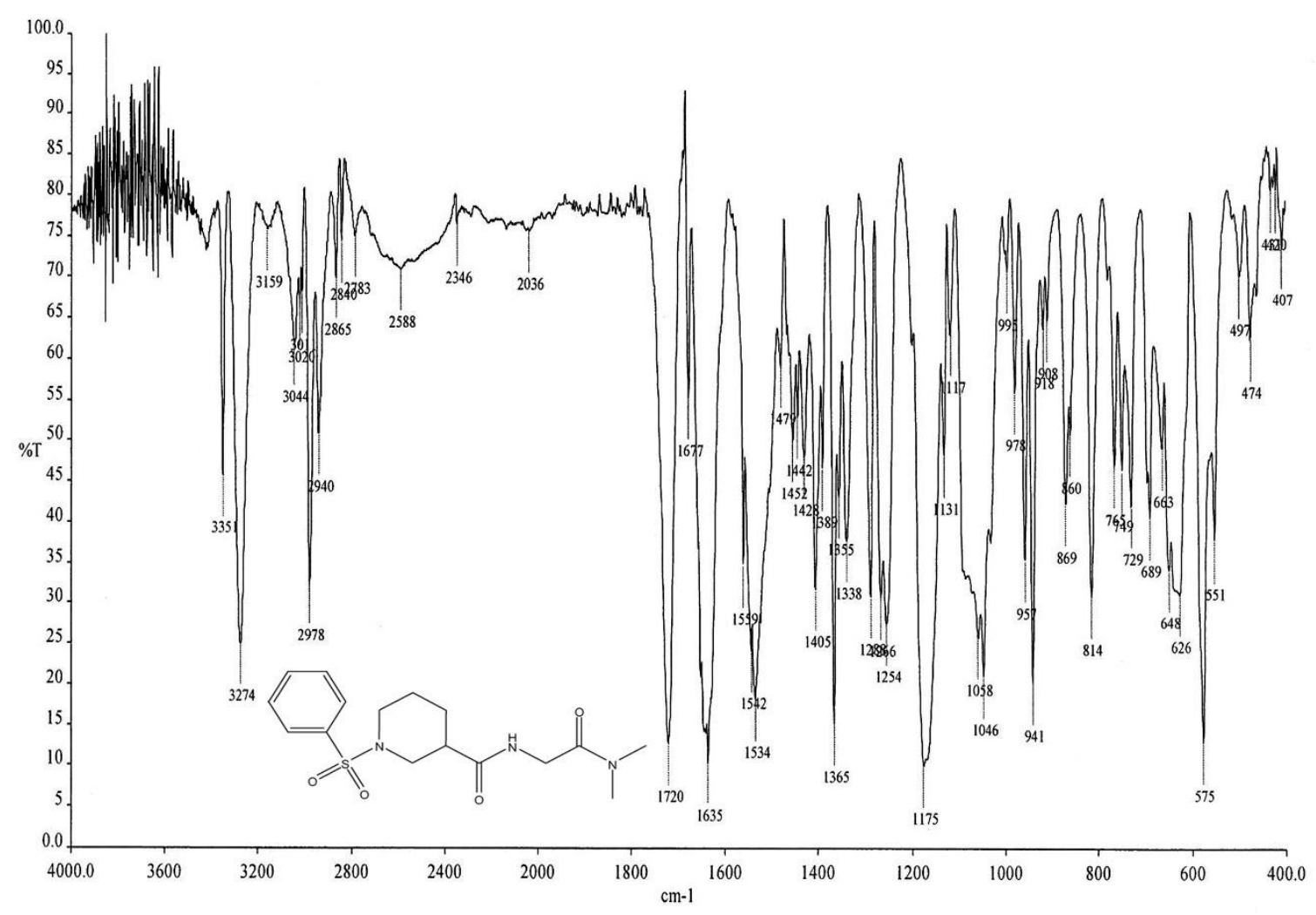

Figure 25: IR of N-(2-(dimethylamino)-2-oxoethyl)-1-(phenylsulfonyl)piperidine-3-carboxamide $\mathbf{6 g}$

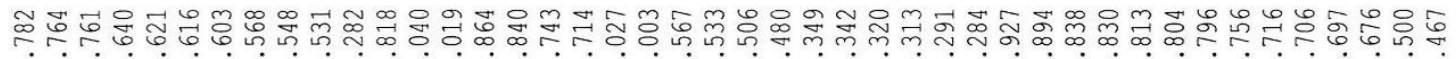

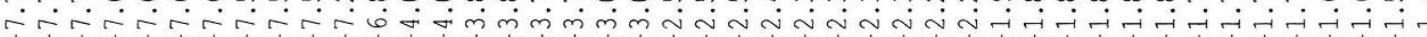

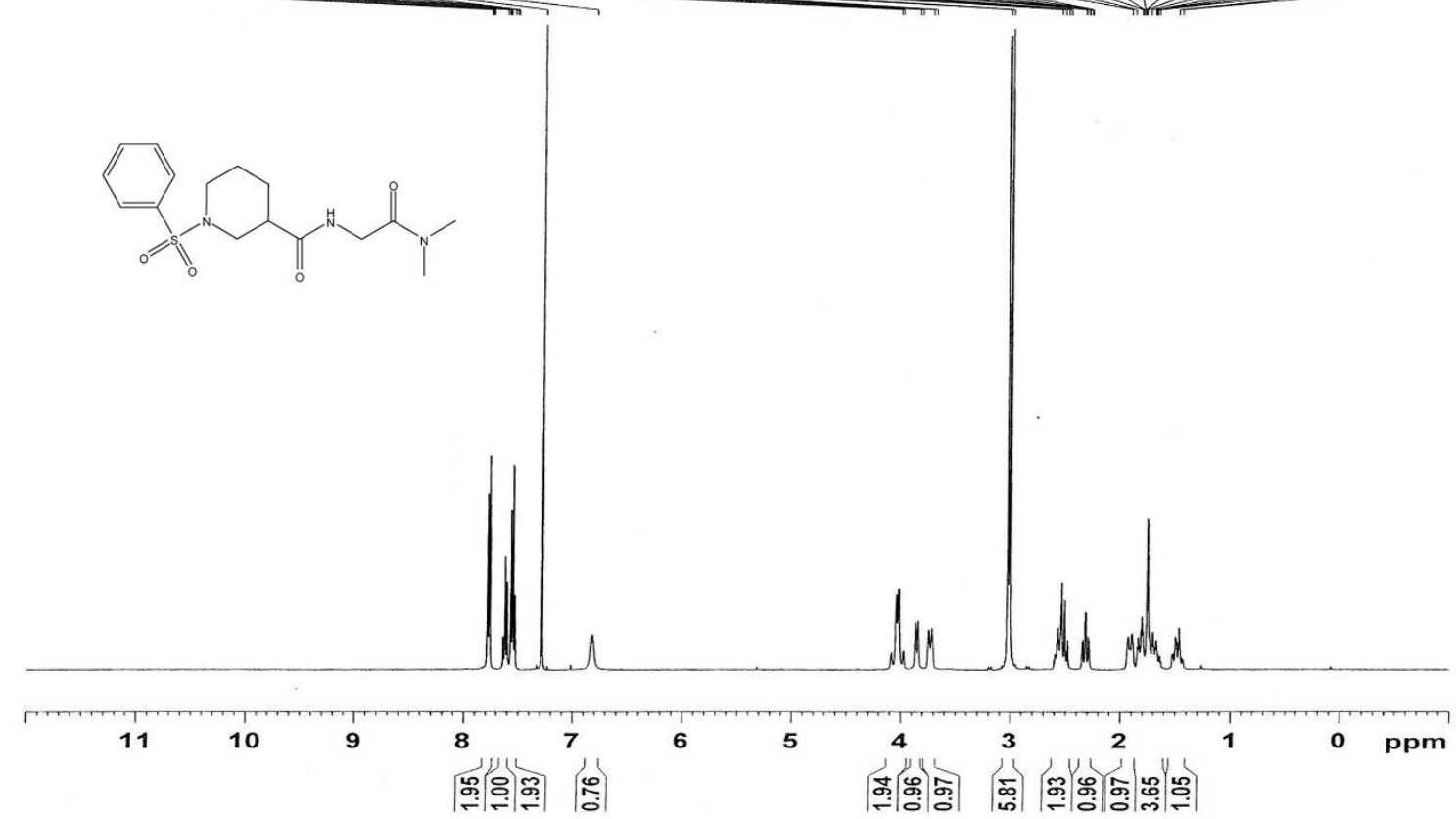

Figure 26: ${ }^{1} \mathrm{H}$ NMR of N-(2-(dimethylamino)-2-oxoethyl)-1-(phenylsulfonyl)piperidine-3-carboxamide $\mathbf{6 g}$ 


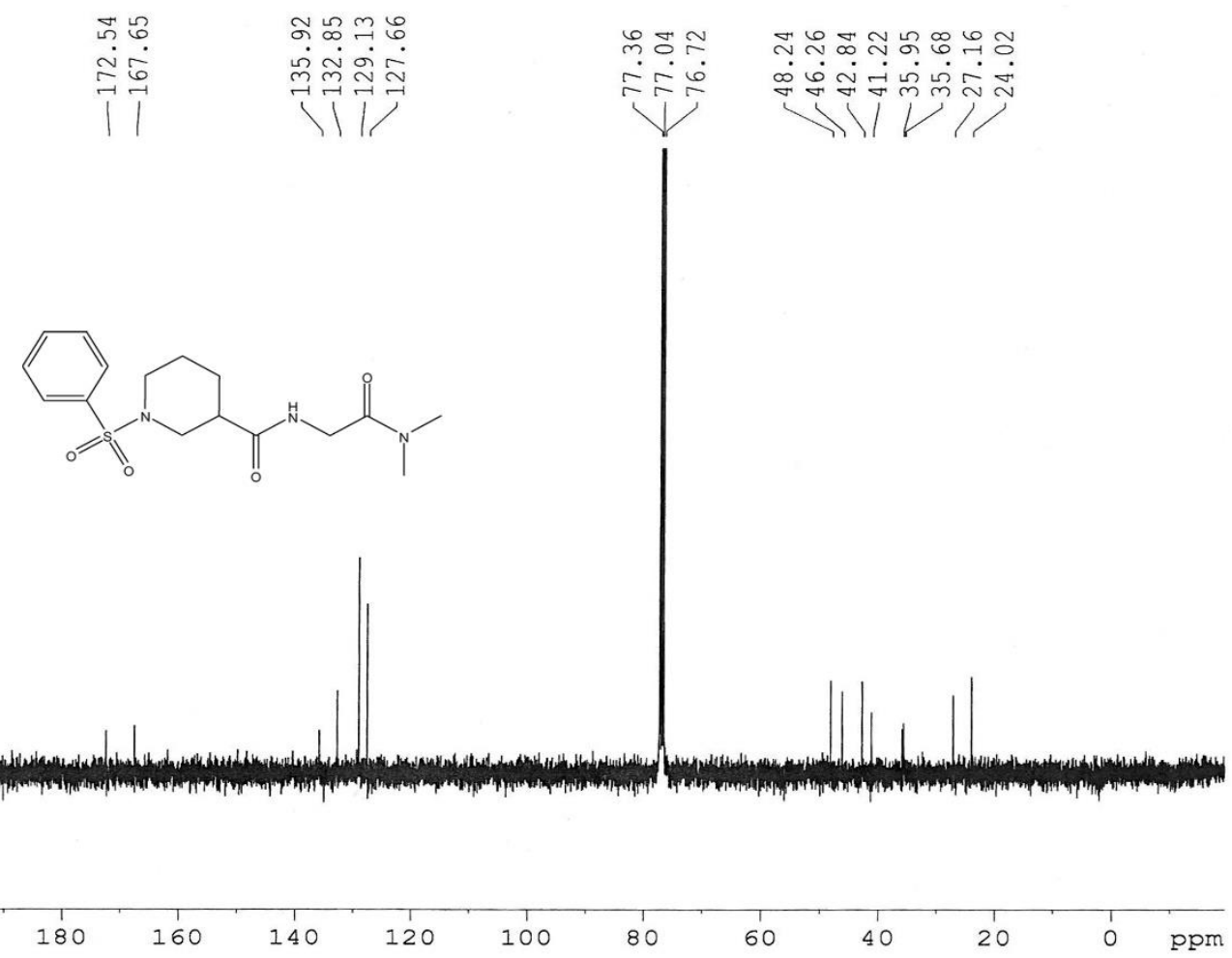

Figure 27: ${ }^{13} \mathrm{C}$ NMR of N-(2-(dimethylamino)-2-oxoethyl)-1-(phenylsulfonyl)piperidine-3-carboxamide $\mathbf{6 g}$

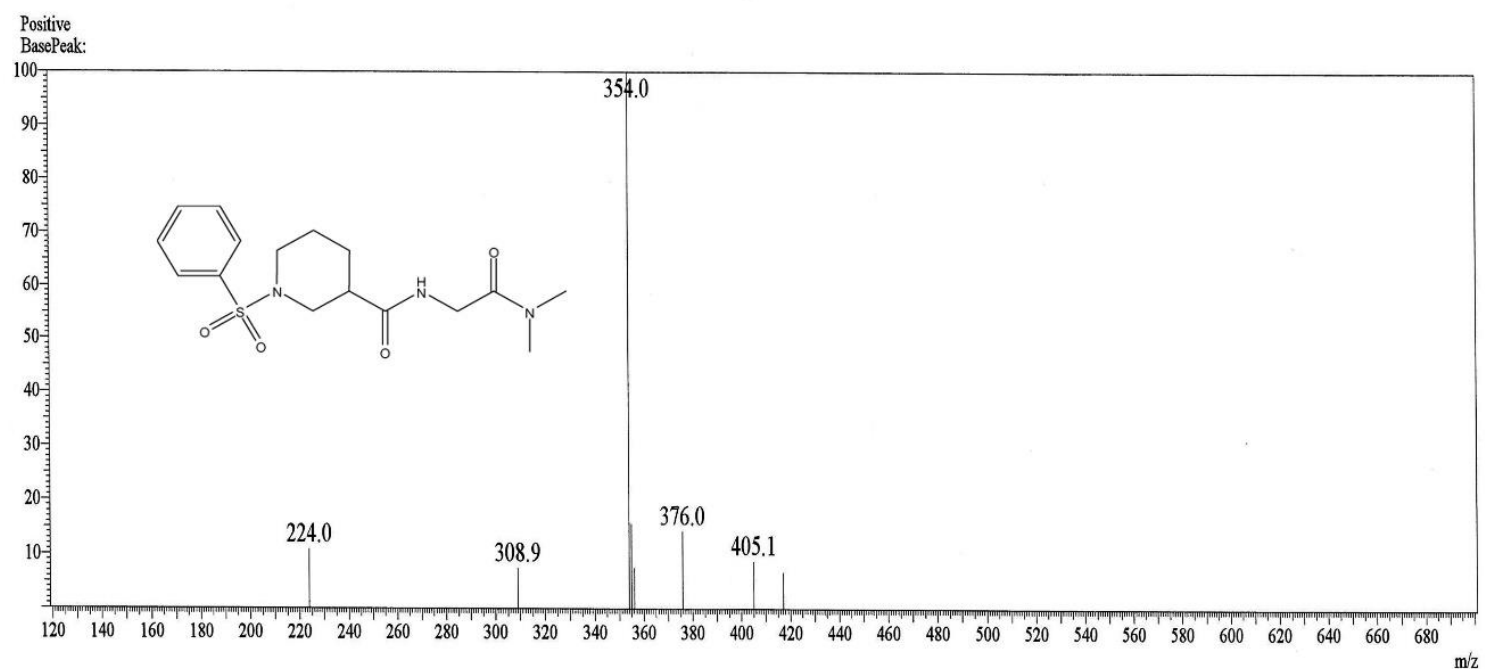

Figure 28: ESI-MS of N-(2-(dimethylamino)-2-oxoethyl)-1-(phenylsulfonyl)piperidine-3-carboxamide $\mathbf{6 g}$ 


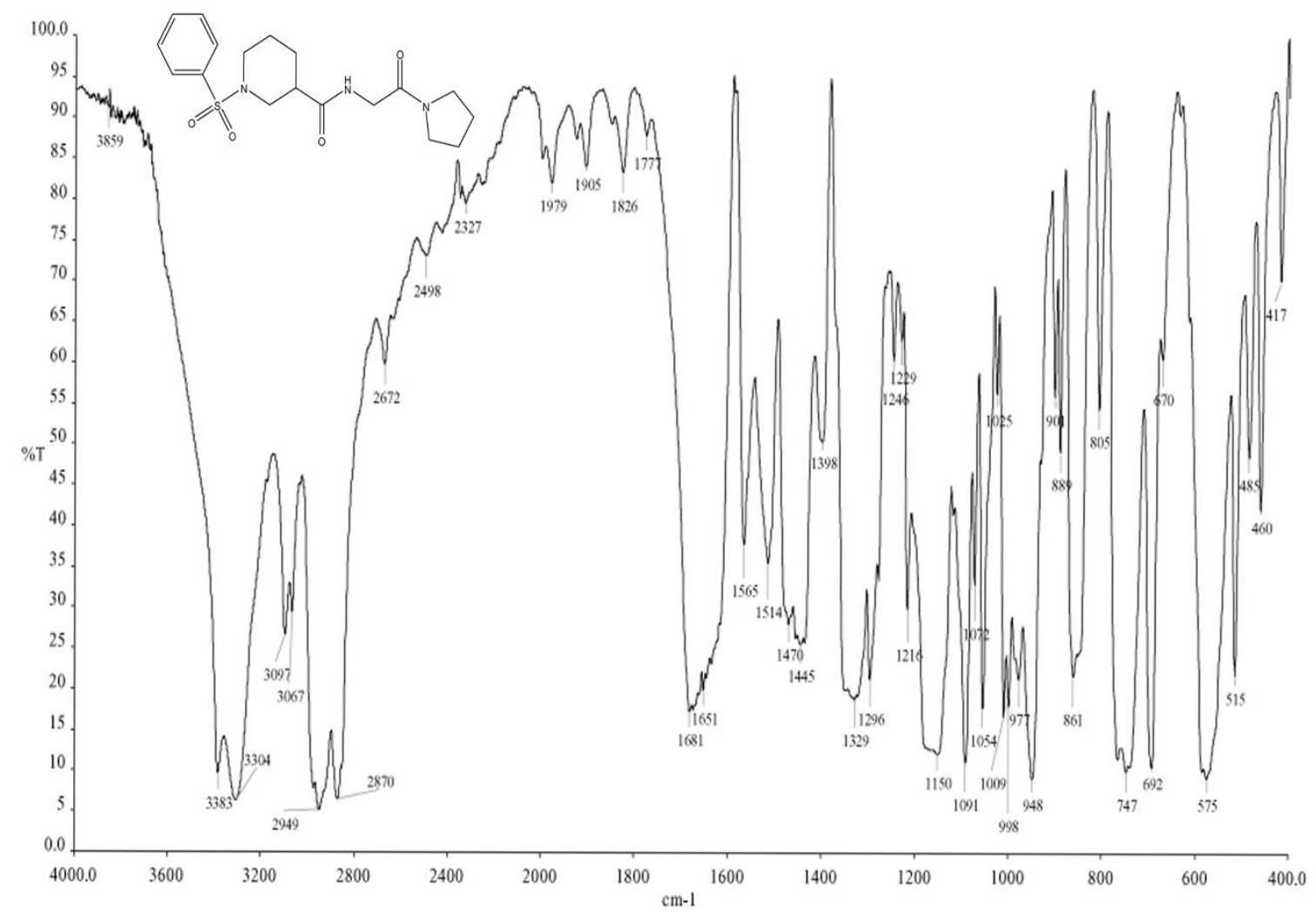

Figure 29: IR of N-(2-oxo-2-(pyrrolidin-1-yl)ethyl)-1-(phenylsulfonyl)piperidine-3-carboxamide $\mathbf{6 h}$

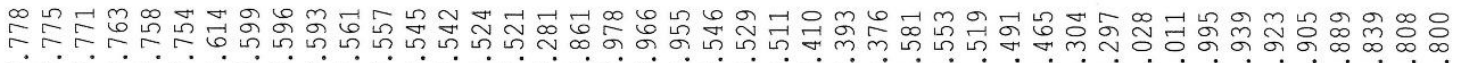

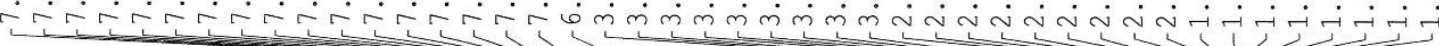

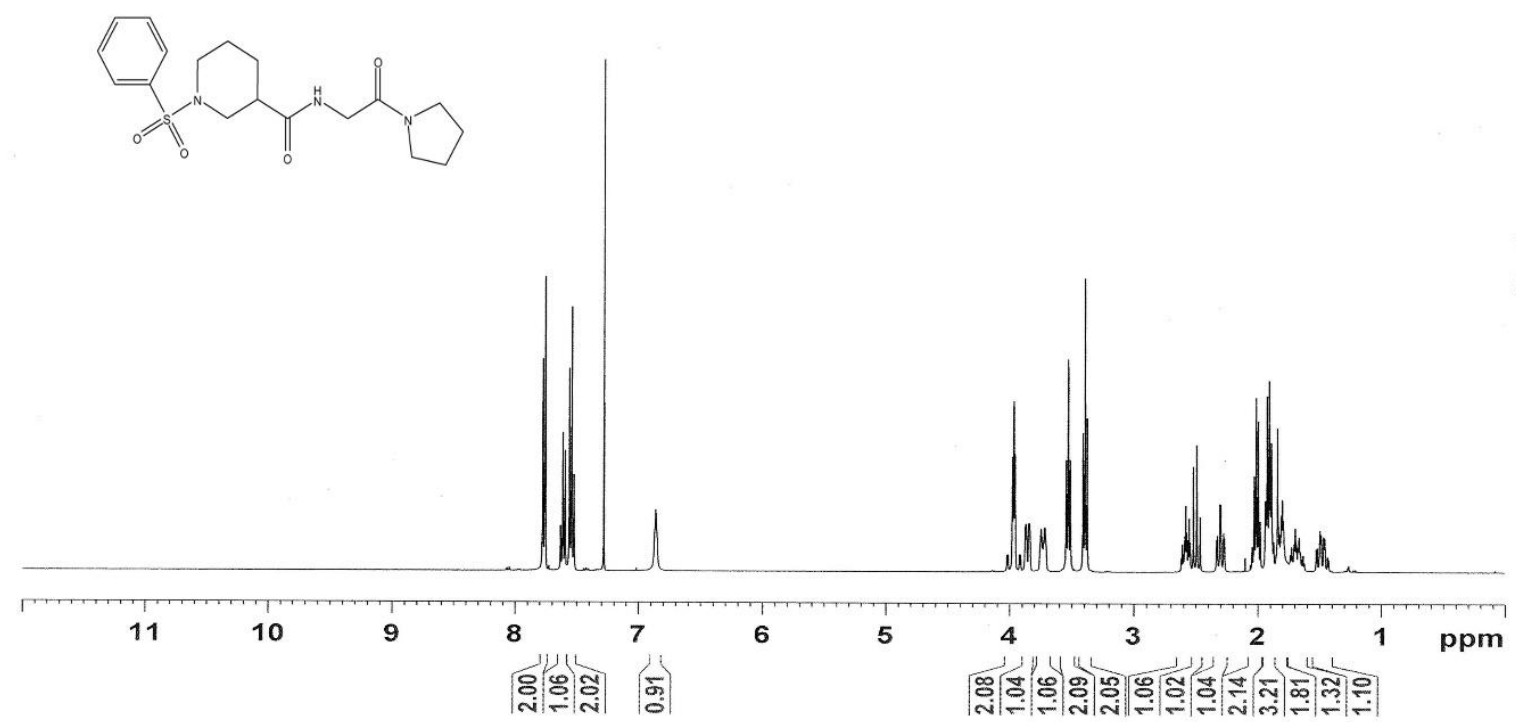

Figure 30: ${ }^{1} \mathrm{H}$ NMR of N-(2-oxo-2-(pyrrolidin-1-yl)ethyl)-1-(phenylsulfonyl)piperidine-3-carboxamide $\mathbf{6 h}$ 


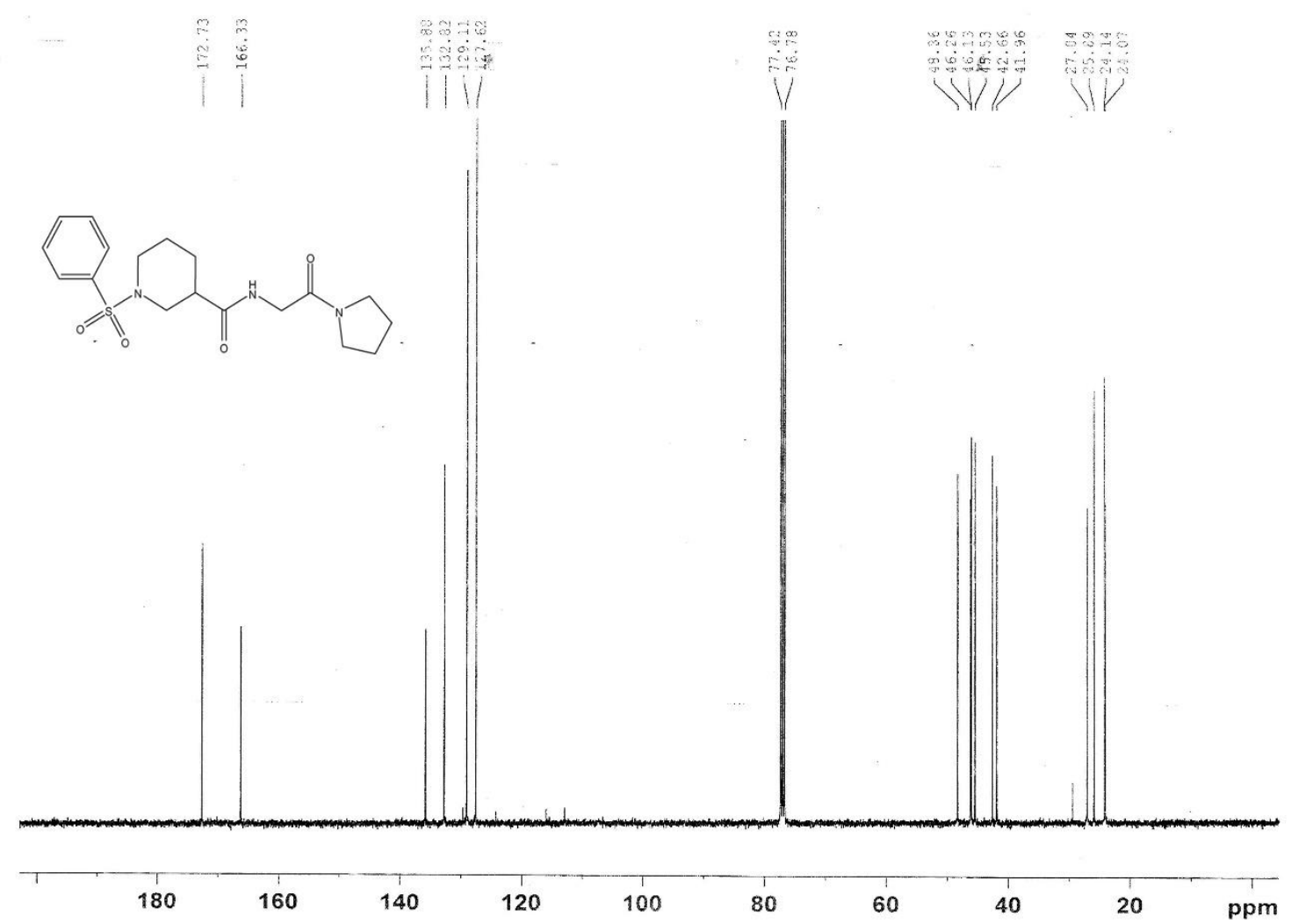

Figure $31:{ }^{13} \mathrm{C}$ NMR of N-(2-oxo-2-(pyrrolidin-1-yl)ethyl)-1-(phenylsulfonyl)piperidine-3-carboxamide $\mathbf{6 h}$

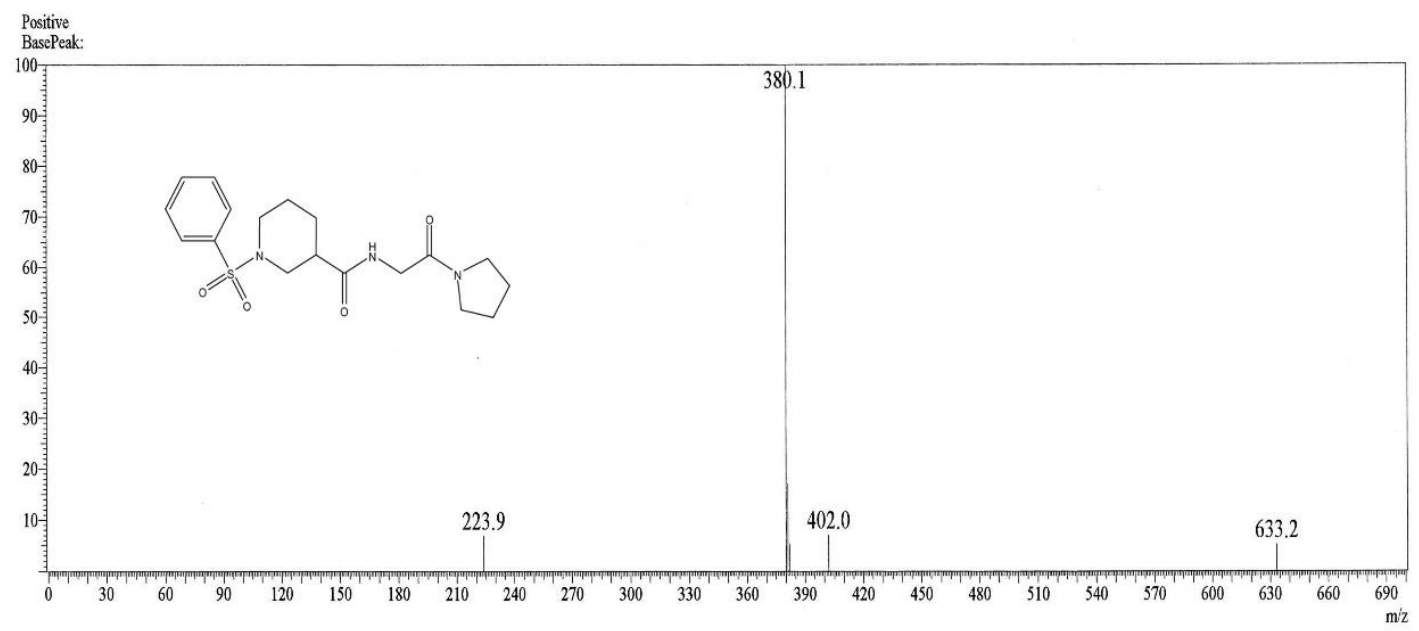

Figure 32: ESI-MS of N-(2-oxo-2-(pyrrolidin-1-yl)ethyl)-1-(phenylsulfonyl)piperidine-3-carboxamide $\mathbf{6 h}$ 


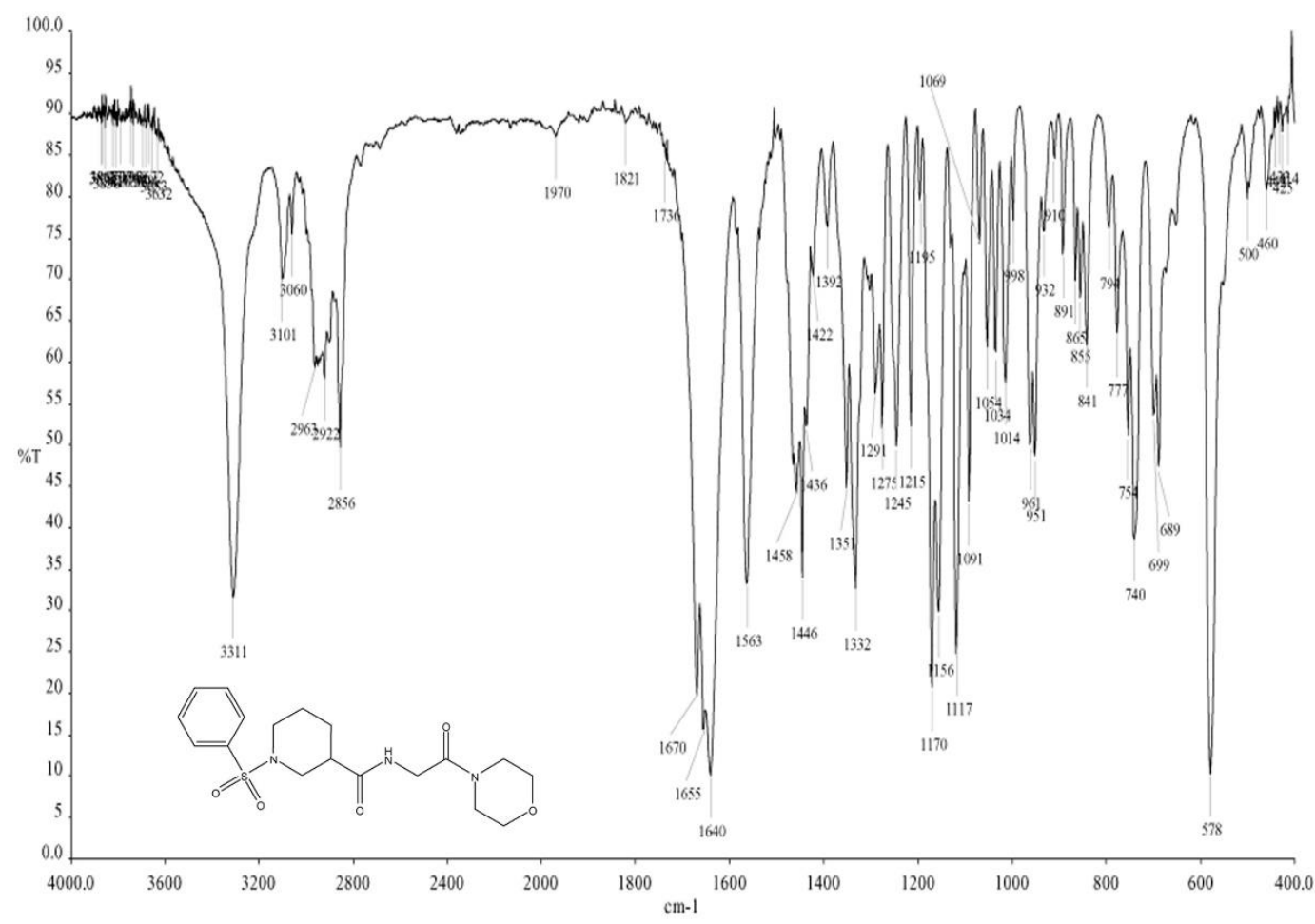

Figure 33: IR of N-(2-morpholino-2-oxoethyl)-1-(phenylsulfonyl)piperidine-3-carboxamide 6i

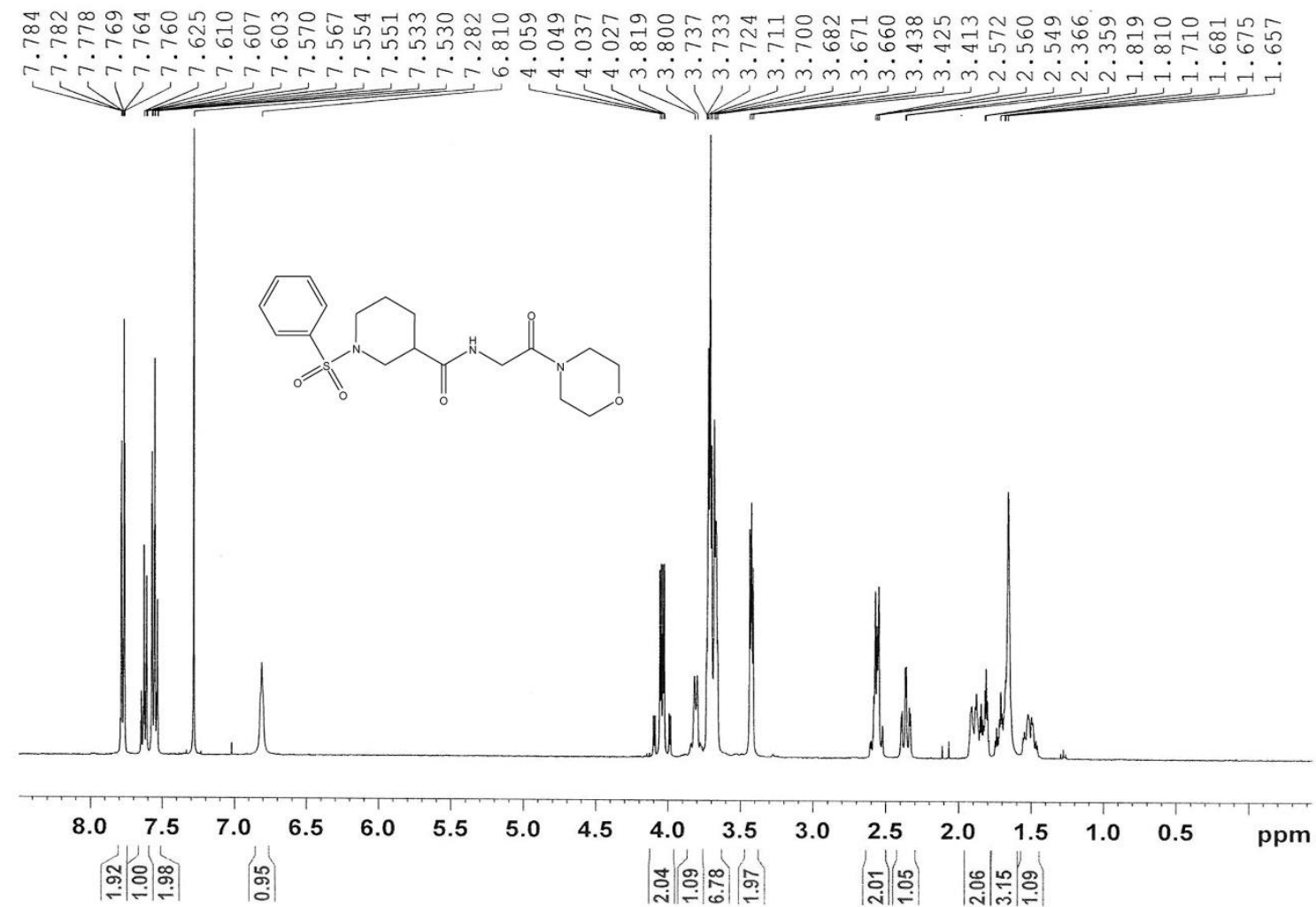

Figure 34: ${ }^{1} \mathrm{H}$ NMR of N-(2-morpholino-2-oxoethyl)-1-(phenylsulfonyl)piperidine-3-carboxamide $6 \mathbf{i}$ 


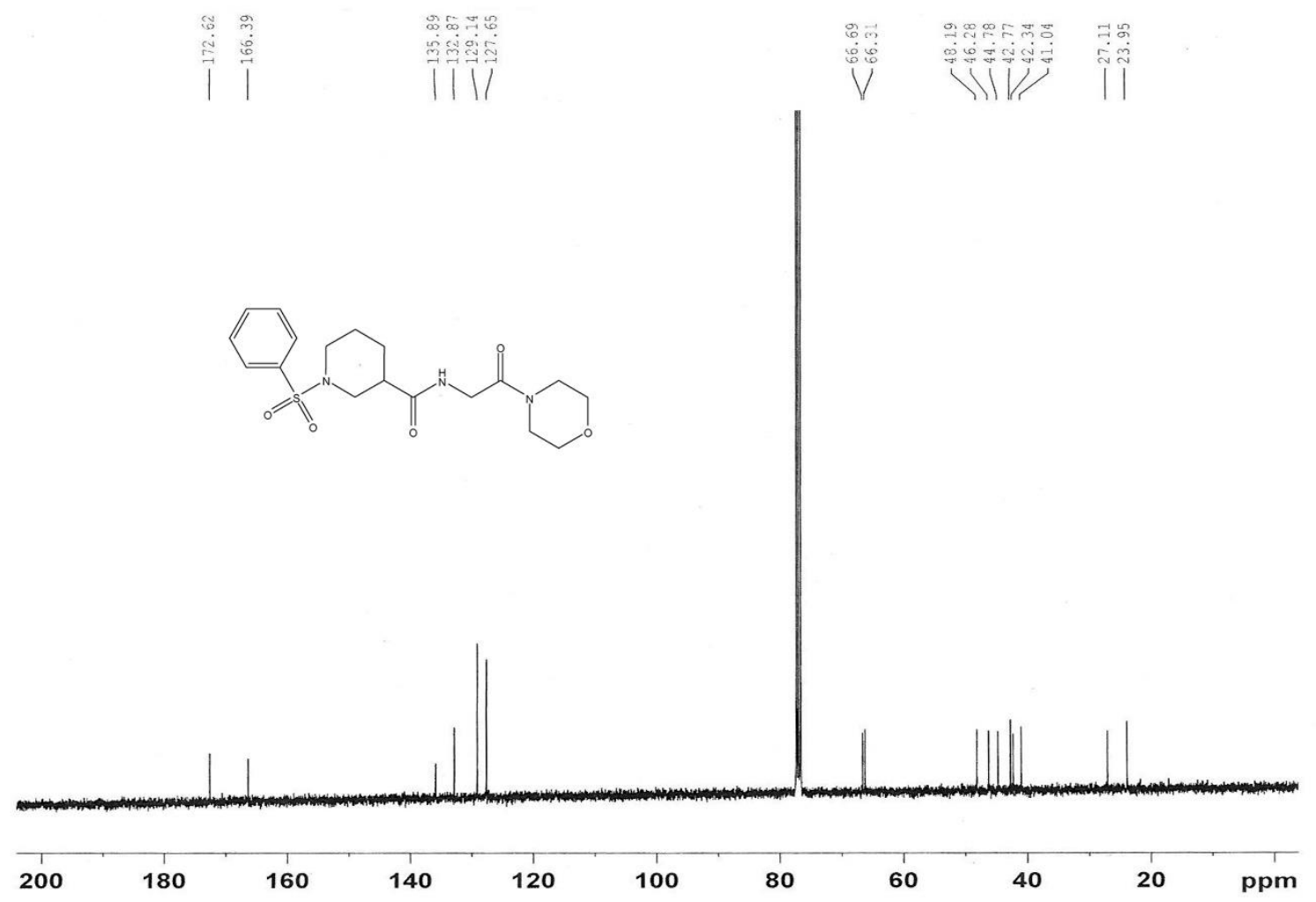

Figure 35: ${ }^{13} \mathrm{C}$ NMR of N-(2-morpholino-2-oxoethyl)-1-(phenylsulfonyl)piperidine-3-carboxamide $6 \mathbf{i}$

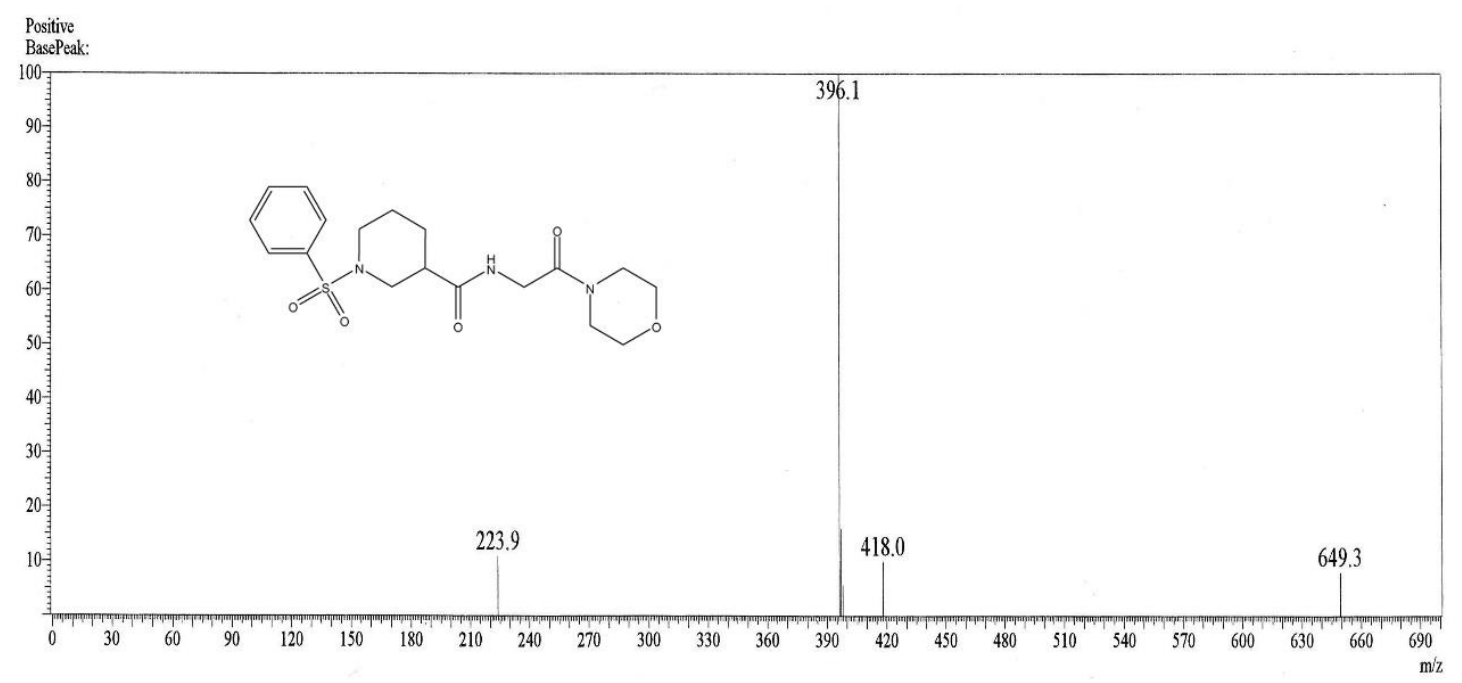

Figure 36: ESI-MS of N-(2-morpholino-2-oxoethyl)-1-(phenylsulfonyl)piperidine-3-carboxamide 6i 


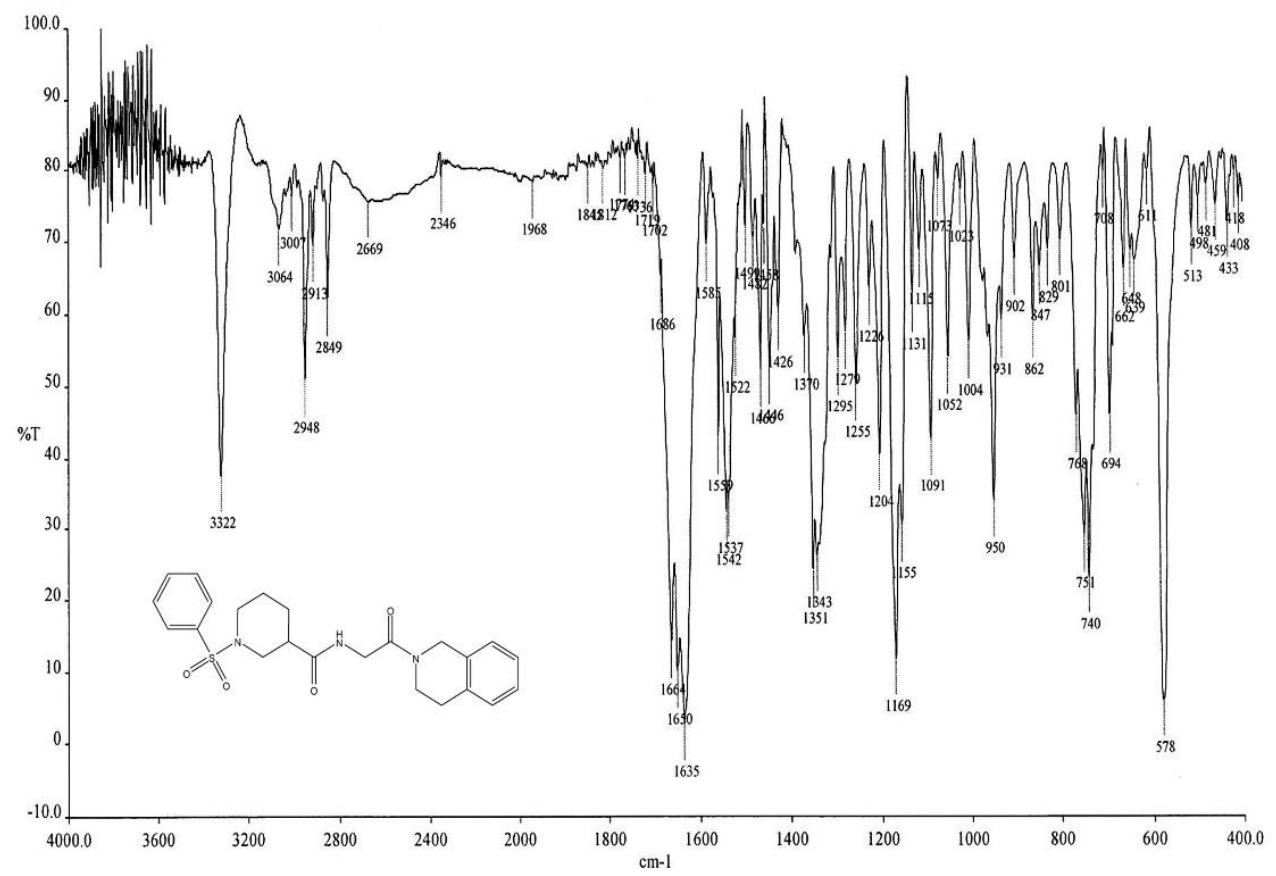

Figure 37: IR of N-(2-(3,4-dihydroisoquinolin-2(1H)-yl)-2-oxoethyl)-1-(phenylsulfonyl)piperidine-3-

carboxamide $\mathbf{6 j}$

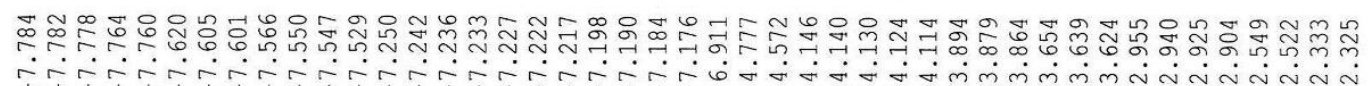

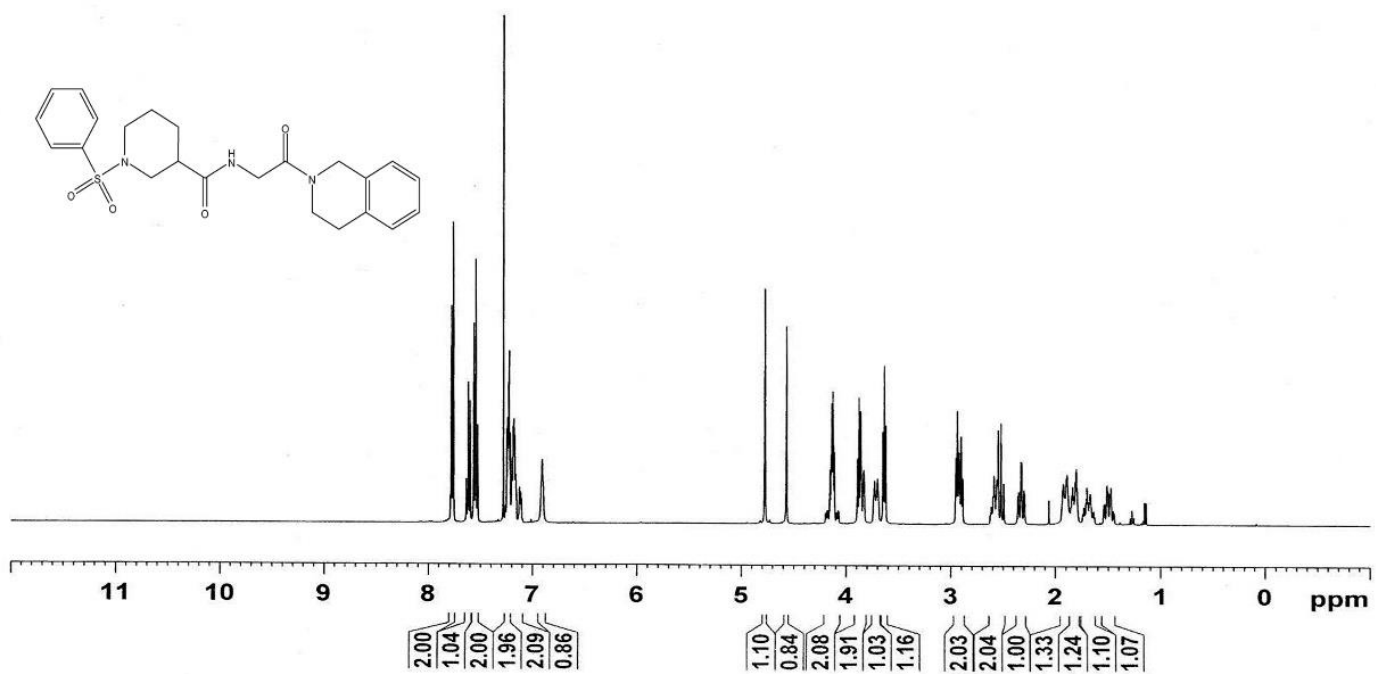

Figure 38: ${ }^{1} \mathrm{H}$ NMR of N-(2-(3,4-dihydroisoquinolin-2(1H)-yl)-2-oxoethyl)-1-(phenylsulfonyl)piperidine-3-

carboxamide $\mathbf{6 j}$ 


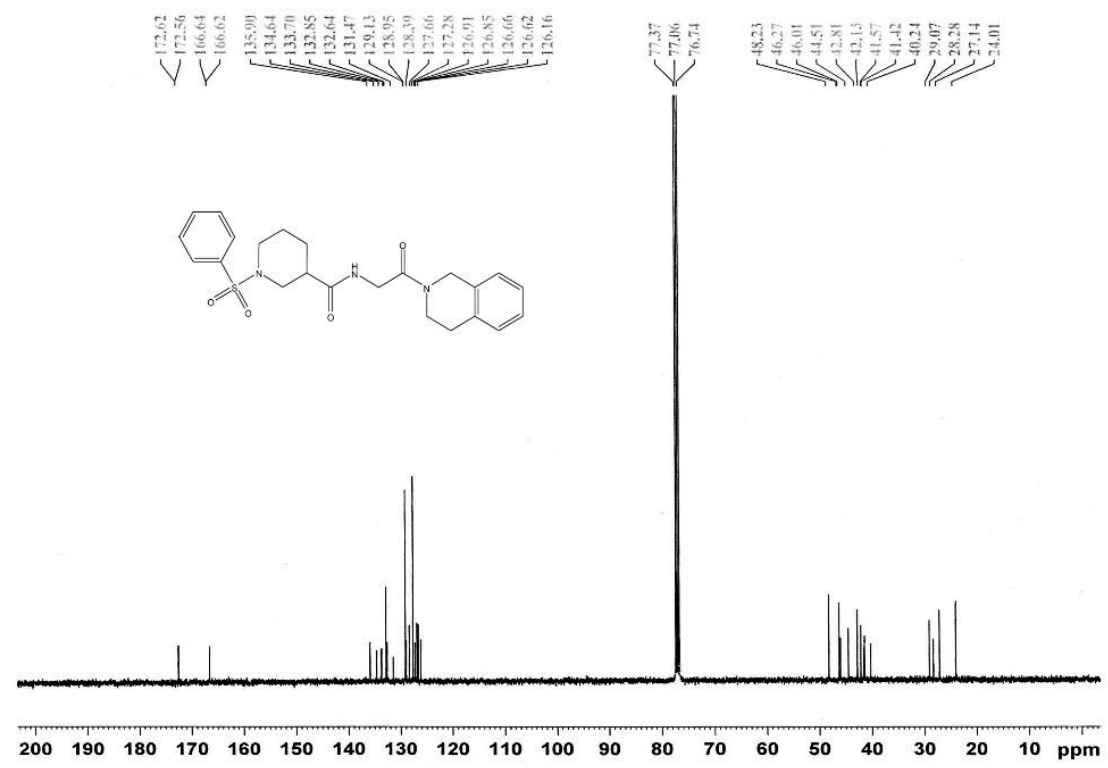

Figure 39: ${ }^{13} \mathrm{C}$ NMR of N-(2-(3,4-dihydroisoquinolin-2(1H)-yl)-2-oxoethyl)-1-(phenylsulfonyl)piperidine-3carboxamide $\mathbf{6 j}$

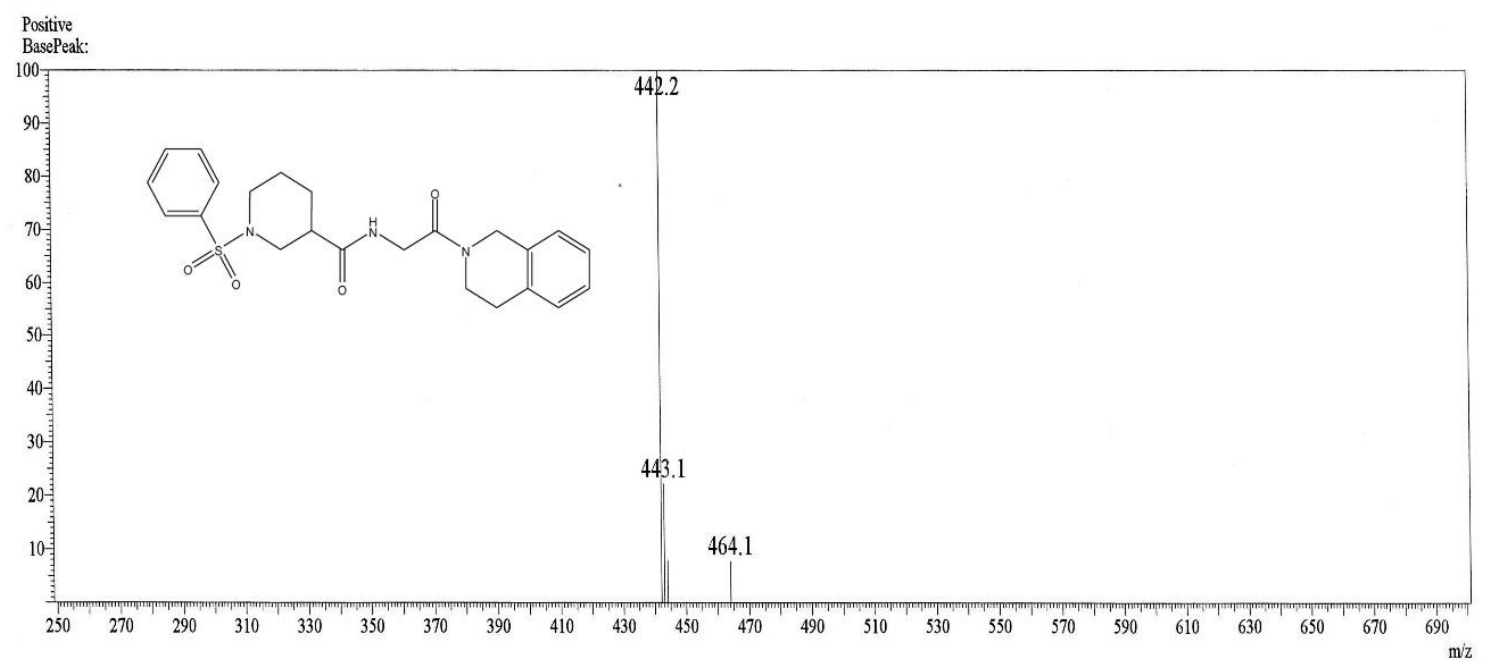

Figure 40: ESI-MS of N-(2-(3,4-dihydroisoquinolin-2(1H)-yl)-2-oxoethyl)-1-(phenylsulfonyl)piperidine-3carboxamide $\mathbf{6 j}$ 


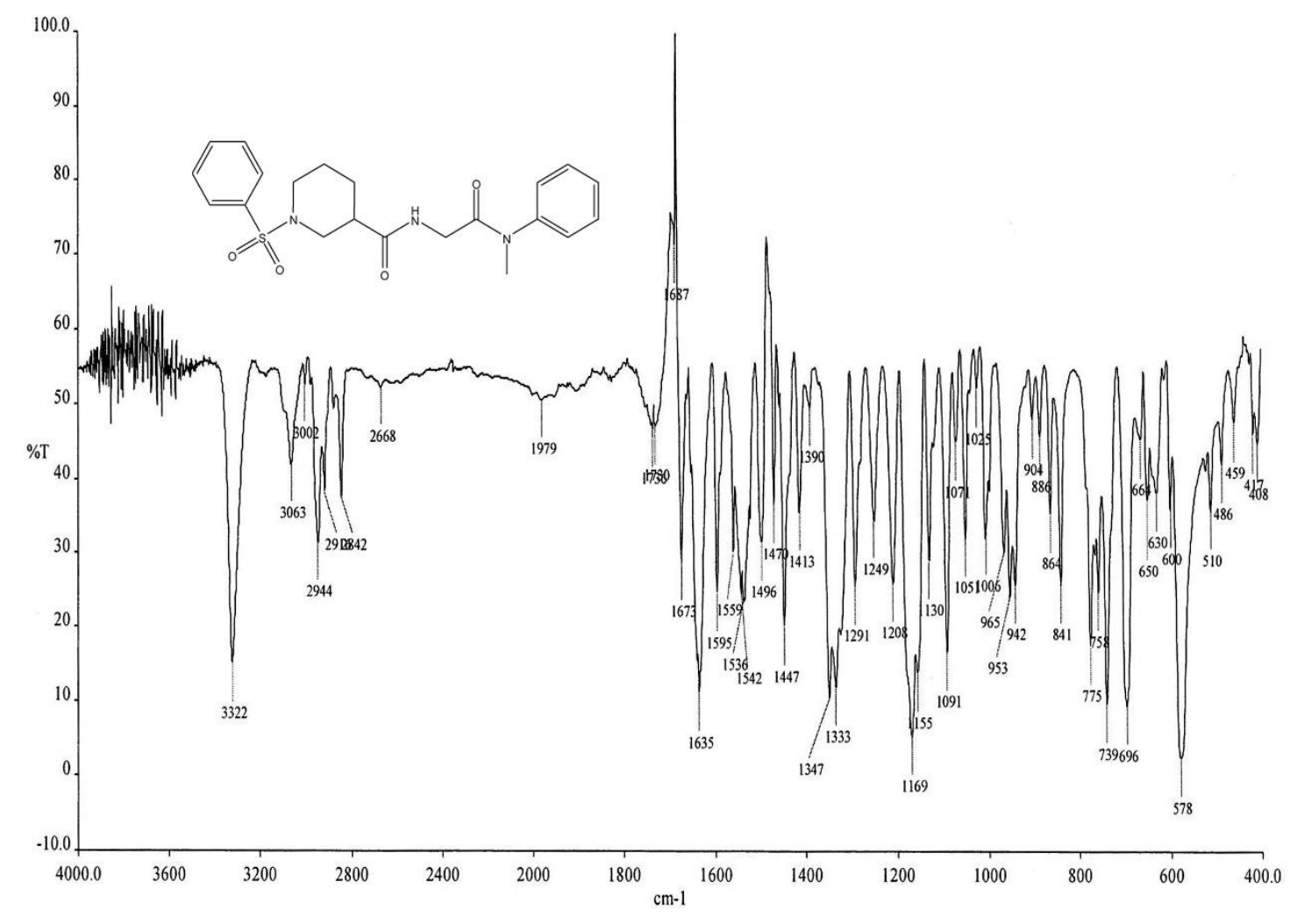

Figure 41: IR of N-(2-(methyl(phenyl)amino)-2-oxoethyl)-1-(phenylsulfonyl)piperidine-3-carboxamide $\mathbf{6 k}$

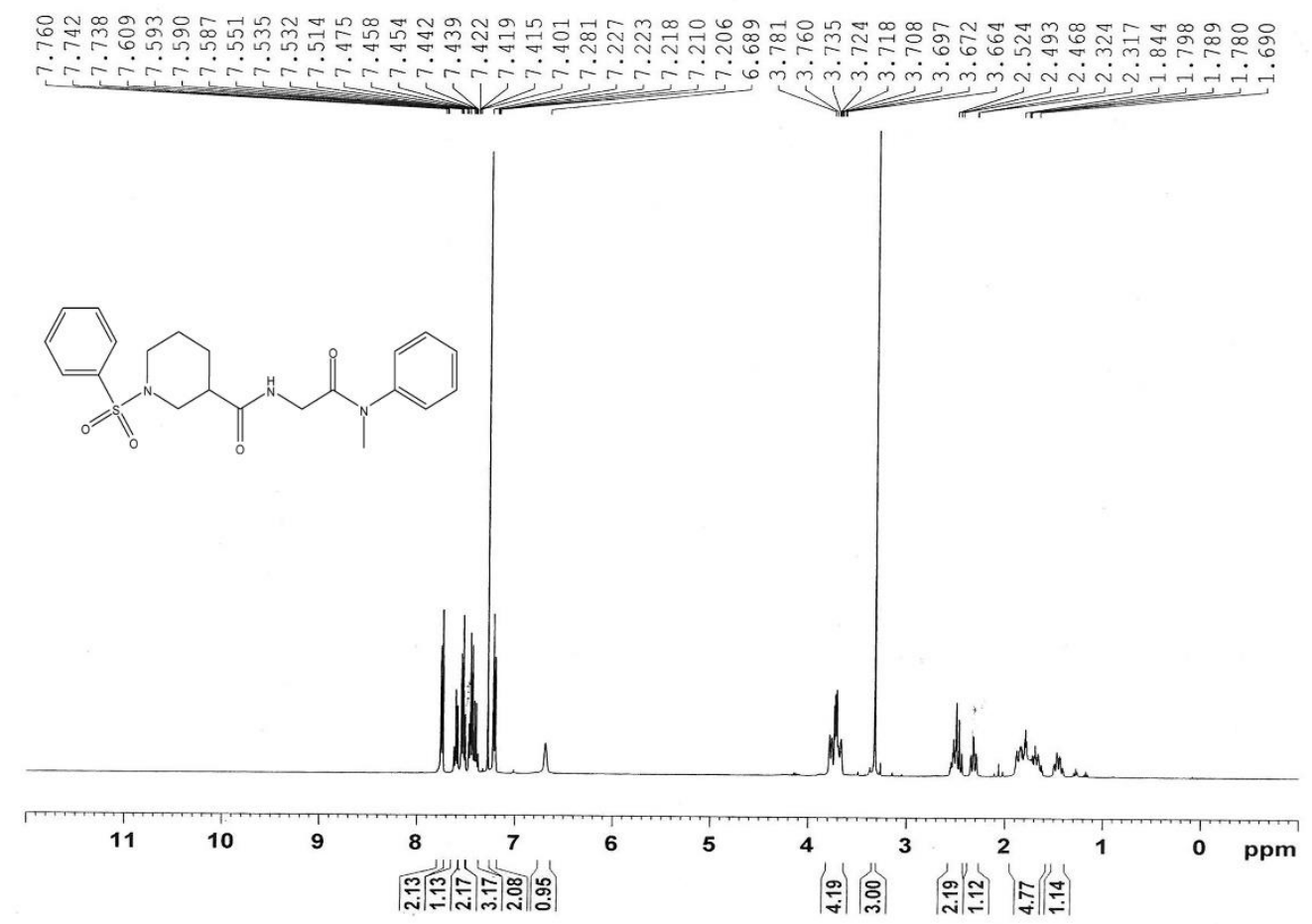

Figure 42: ${ }^{1} \mathrm{H}$ NMR of N-(2-(methyl(phenyl)amino)-2-oxoethyl)-1-(phenylsulfonyl)piperidine-3-carboxamide 


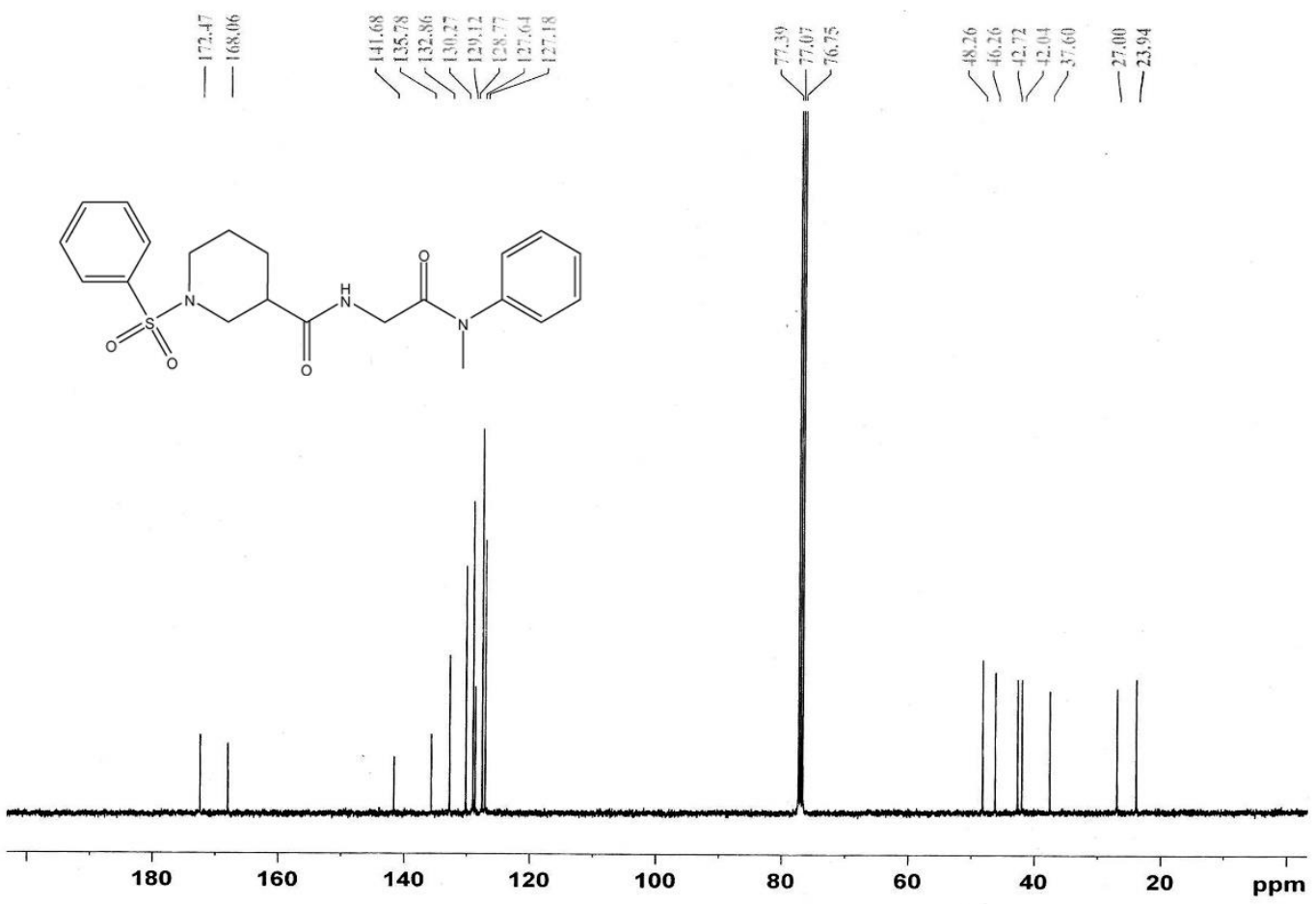

Figure $43:{ }^{13} \mathrm{C}$ NMR of $\mathrm{N}$-(2-(methyl(phenyl)amino)-2-oxoethyl)-1-(phenylsulfonyl)piperidine-3-carboxamide $6 \mathbf{k}$

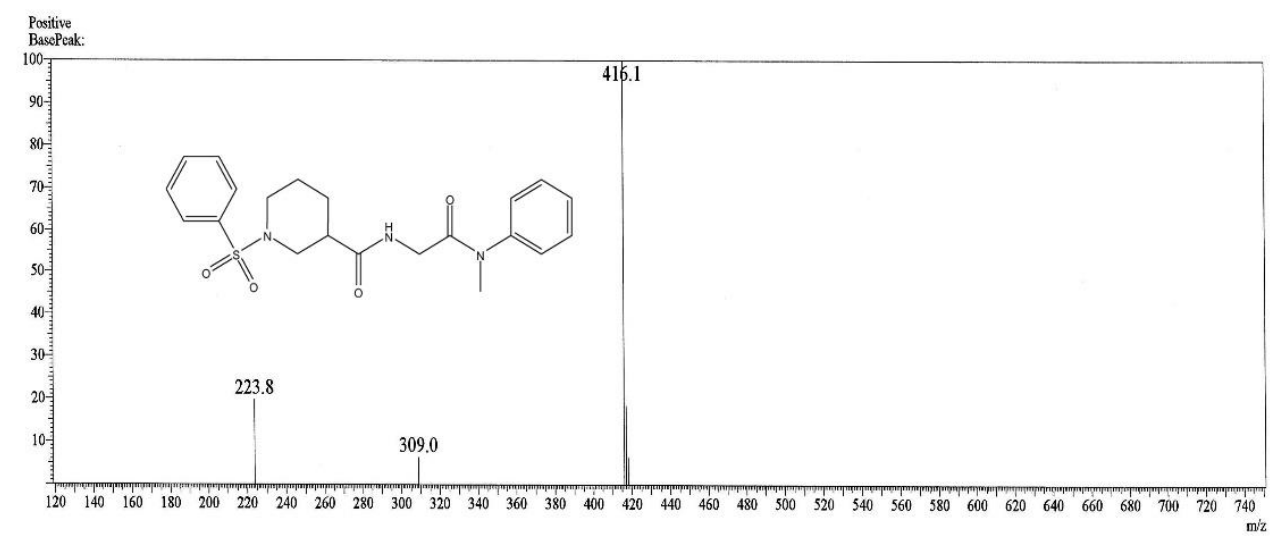

Figure 44: ESI-MS of N-(2-(methyl(phenyl)amino)-2-oxoethyl)-1-(phenylsulfonyl)piperidine-3-carboxamide 6k 